\title{
Microstructural and Micromechanical Characterization of IN718 Theta Shaped Specimens Built with Electron Beam Melting
}

\author{
Ercan Cakmak $^{a^{*}}$, Michael M. Kirka ${ }^{\text {a,b }}$, Thomas R. Watkinsa, Ryan C. Cooper ${ }^{\mathrm{a}}$, Ke An ${ }^{\mathrm{c}}$, Hahn Choo ${ }^{\mathrm{d}}$, Wei \\ $W_{u^{c}}$, Ryan R. Dehoff $f^{a, b}$ and Sudarsanam S. Babu ${ }^{\mathrm{d}, \mathrm{e}, \mathrm{f}}$
}

${ }^{a}$ Materials Science and Technology Division, Oak Ridge National Laboratory, Oak Ridge, TN 37831, USA

${ }^{b}$ Manufacturing Demonstration Facility, Oak Ridge National Laboratory, Knoxville, TN 37932, USA

${ }^{c}$ Chemical and Engineering Materials Division, Oak Ridge National Laboratory, Oak Ridge, TN 37831, USA

${ }^{d}$ Department of Materials Science and Engineering, The University of Tennessee, Knoxville, TN 37996, USA

${ }^{e}$ Energy \& Transportation Science Division, Oak Ridge National Laboratory, Oak Ridge, TN 37831, USA

${ }^{f}$ Department of Mechanical, Aerospace and Biomedical Engineering, The University of Tennessee, Knoxville, TN 37996, USA

\section{*Corresponding Author: \\ Ercan Cakmak \\ Materials Science and Technology Division \\ Oak Ridge National Laboratory ${ }^{\#}$ \\ Oak Ridge, TN 37831-6064}

Tel: (865) 574-6538

E-mail: cakmake@ornl.gov

* Author to whom correspondence should be addressed; electronic mail: cakmake@ornl.gov

\footnotetext{
\# This manuscript has been authored by UT-Battelle, LLC under Contract No. DE-AC05-00OR22725 with the U.S. Department of Energy. The United States Government retains and the publisher, by accepting the article for publication, acknowledges that the United States Government retains a non-exclusive, paid-up, irrevocable, world-wide license to publish or reproduce the published form of this manuscript, or allow others to do so, for United States Government purposes. The Department of Energy will provide public access to these results of federally sponsored research in accordance with the DOE Public Access Plan (http://energy.gov/downloads/doe-public-access-plan ).
} 


\begin{abstract}
Theta-shaped specimens were additively manufactured out of Inconel 718 powders using an electron beam melting technique, as a model complex load bearing structure. Two different build strategies were employed; producing two sets of specimens. Microstructural and micromechanical characterizations were performed using electron back-scatter, synchrotron x-ray and in-situ neutron diffraction techniques. In particular, the cross-members of the specimens were the focus of the synchrotron $x$-ray and in-situ neutron diffraction measurements. The build strategies employed resulted in the formation of distinct microstructures and crystallographic textures, signifying the importance of build-parameter manipulation for microstructural optimization. Large strain anisotropy of the different lattice planes was observed during in-situ loading. Texture was concluded to have a distinct effect upon both the axial and transverse strain responses of the cross-members. In particular, the (200), (220) and (420) transverse lattice strains all showed unexpected overlapping trends in both builds. This was related to the strong $\{200\}$ textures along the build/loading direction, providing agreement between the experimental and calculated results.
\end{abstract}

Keywords: Additive manufacturing; Nickel-based superalloys; Neutron diffraction; Texture; Lattice strains 


\section{Introduction}

Additive Manufacturing (AM) techniques have gained significant attention in the past decade with the new perspective they offer on manufacturing. With such techniques, the finished product is made additively from raw feedstock materials such as powders, wires or tapes [1-3], as opposed to conventional subtractive techniques that rely on removal of material from bulk monoliths.

In powder-bed AM technologies, the build proceeds by raking a fine layer (typically $\sim 50-100 \mu \mathrm{m}$ high) of the metal powder over a base plate on the build table. Subsequently, a directed heat source, such as an electron or laser beam, locally fuses the powder to the base plate and later to the prior fused material. The actual fusing itself may be accomplished in a variety of patterns in concert with varied heat source, speed and energy. By its nature, powder-bed technologies offer more freedom in design and potential for more control over the build parameters thus affecting the final build characteristics such as grain morphology and orientations, modulus, strength and ductility, etc. Because of such versatility, powder-bed based AM techniques have gathered considerable attention [1, 3-14].

So far, the main focus of AM has been obtaining structures free of macroscopic defects such as porosity, shrinkage or cracks, and with good dimensional tolerances. However, due to the rapid melt-quench nature of these techniques, analogous to welding, and the high temperatures involved, the builds suffer from effects such as residual stresses, strong preferred orientations and large grains. Particularly, the as-built residual stresses pose a major concern. However, parts built with the electron beam melting $(\mathrm{EBM})$ technique do not suffer as much from residual 
stresses as does the laser based techniques [14] due to the higher build-chamber temperatures (at the order of $0.7 \times \mathrm{T}_{\mathrm{M}}$ ) which helps to anneal/stress-relive the sample during the build.

During the AM process, multiple cycles of melting and solidification take place at relatively high temperatures resulting in grain growth. Additionally, the grain growth can be significantly influenced by the crystallographic orientations as growth can be favored along a preferred crystallographic direction. For FCC alloys, the most favorable directions for growth are the $\langle 100\rangle$ directions [15-17]. Therefore, grains with one of their $<100\rangle$ directions aligned with the direction of heat flow will be favored over the others in a polycrystalline specimen [16]. Accordingly, the finished products are often accompanied by strong crystallographic textures.

Furthermore, in the powder based AM, when a new layer of powder is raked over the already deposited material, the fusing sequence melts some portion of the previously deposited layers along with the free powder. While this bonds the new and existing layers, it also enables the extension of the crystallographic orientations [12] as the partial remelting of the previously built layers stimulates the formation of an epitaxy between the layers $[4,6,15,18]$. Such epitaxy is also responsible for the partially unidirectional columnar grain growth in additively manufactured parts.

The columnar grain growth is also a direct consequence of the thermal gradient that exists along the build direction. That is, during the deposition process, the already deposited material acts as a heat sink while the loose powder in the powder bed surrounding the build forms an insulating envelope. Thus, the heat flow is mainly dominated from the top of the build towards the build 
plate, i.e., along the build direction [15]. For instance, one of the most studied alloys systems with $\mathrm{AM}$ is the nickel-base superalloys and overall, the formation of columnar grains with their (200) plane normals parallel to the build direction has frequently been reported for these systems $[4,6,7,12,15,19]$ with some grains several hundreds of $\mu \mathrm{m}$ long.

However, the versatile nature of powder-bed AM can enable tailoring of the microstructures through adjustment of the build parameters. For example, by intelligently manipulating the build parameters, the $\langle 100\rangle$ preferred growth direction of the grains can be aligned along different sample directions in each layer to create a more random/isotropic build. For instance, in their preliminary study Dehoff et al. [20] has reported successfully manipulating the morphology and textures of EBM built IN718 parts through alteration of the build parameters including the beam path, beam speed and the beam current. Through various combinations of beam parameters, Dehoff et al. [20] have managed to obtain various structures from columnar grains with very strong textures to near equiaxed grains with weaker textures. Körner et al. has also reported achieving various grain morphologies, i.e., columnar or equiaxed, through tuning the build parameters in an IN718 alloy built using EBM [21]. Similarly, by dynamically manipulating the electron beam current (I) and electron beam velocity (v) Dehoff et al. [22] reported adjusting the melt pool shape, size, temperature gradient $(\mathrm{G}, \mathrm{K} / \mathrm{m})$ and liquid-solid interface velocity $(\mathrm{R}, \mathrm{m} / \mathrm{s})$ parameters during the build sequence to obtain regions with columnar, equiaxed and mix grain morphologies within the same build. Such studies demonstrate the promise powder-bed AM techniques hold in terms of micro-engineering the build structures and the importance of continued research in this front. 
While the underlying premise of AM technologies is to allow direct fabrication of complex structures, current AM literature is mainly focused on simple-shaped test builds $[4-6,9,10,13$, $19,23,24]$. However, studies of more complex shapes are now needed to advance the state-ofthe-art. Here, theta shaped specimens [25, 26], named after the physical resemblance to the Greek letter “ $\Theta$ ”, can act as model complex load-bearing systems with interconnecting arches and beams commonly encountered in real-life engineering components. In-situ testing of such load-bearing structures using neutron diffraction [27-29] can offer valuable information regarding deformation at the granular level. By probing the deformation response of various (hkl) grain families, the anisotropic distribution of lattice strains can be obtained. Such information is needed for a fundamental understanding of the micro-mechanical behavior of AM parts. However, to date in-situ loading behavior of AM parts has not been studied in much detail.

In our recent work [30], two sets of theta shaped specimens were built out of IN718; employing two different build strategies using the EBM technique. This work served to validate the mechanical stability and structural integrity of this model geometry through various mechanical tests (both ex-situ and in-situ) and analyses using Digital Image Correlation (DIC) and Finite Element Modeling (FEM) [30]. In the current work, the effects of the build strategies on the microscopic and micro-mechanical properties of the theta shaped specimens are studied where Electron Back-Scatter Diffraction (EBSD) is used to probe the grain morphologies and local grain orientations; Synchrotron X-ray Diffraction (SXRD) is used to obtain the bulk textures and in-situ neutron diffraction is used to study the micro-mechanical behavior. The effects of the build strategies on the observed microstructures are discussed in terms of grain morphologies and preferred orientations. The micro-mechanical behavior is discussed in terms of anisotropic 
distribution of lattice strains and load partitioning mechanisms. Additionally, the influence of texture on the observed lattice strain evolutions is discussed.

\section{Experimental details}

\subsection{Sample fabrication with EBM}

Two sets of five IN718 theta specimens were built using the Arcam EBM technique at ORNL's Manufacturing Demonstration Facility (MDF) with an Arcam S12 machine ${ }^{1}$. The composition of the gas atomized IN718 powder (with spherical morphology) used for the build was 18.2-Fe, 18.2-Cr, 3-Mo, 5-Nb, 1-Ti, 0.5-Al, 0.1-Co, 0.1-Mn, 0.05-Cu, 0.2-Si, 0.05-C(max) and balance $\mathrm{Ni}$; in wt.\%; with a particle size distribution of 40-125 $\mu \mathrm{m}$.

Before the start of the build, the build plate was heated to a temperature of $975^{\circ} \mathrm{C}$ and held for 30 minutes to allow the temperature to stabilize throughout the powder bed. One set of specimens was built with a beam scan pattern that manipulated the electron beam to result in a spot heat source. The second set of specimens was built using Arcam's standard commercially available melt theme for IN718 that treats the electron beam as a line heat source. The build sequence schematics for the spot and line builds are presented in Figure 1a and b, respectively. The spot heat source scheme consisted of melting the material one spot at a time in a pattern to control the bulk heat input according to the scheme described by Kirka [31]. The line heat source scheme on the other hand, consisted of a snake raster pattern that was rotated $90^{\circ}$ after each new layer.

\footnotetext{
${ }^{1}$ Arcam AB, Krokslätts Fabriker 27A, SE431 37 Mölndal, Sweden, www.arcam.com.
} 
The build direction of the samples was parallel to the long axis of the cross-member (see axial direction in Figure 3). A layer thickness of $50 \mu \mathrm{m}$ was used for both builds and the total build process took 24 hours with an average time per layer of 70 seconds. The samples were allowed to cool for 7.2 hours under vacuum and no post-process heat treatments were performed. More details about the build parameters employed are presented in [30] (also see the supplementary document).

In order to obtain the axial and transverse lattice strains from the cross-member during the in-situ neutron diffraction experiments, these theta-shaped specimens were designed housing four beam windows (see Figure 3). In terms of sample dimensions, the cross-member was designed to have a $4 \times 4 \mathrm{~mm}^{2}$ cross-sectional area with a total length of $40.4 \mathrm{~mm}$ and gauge length of $28.4 \mathrm{~mm}$. The samples had outer and inner ring diameters of 60 and $44 \mathrm{~mm}$, respectively. The beam windows were $9 \mathrm{~mm}$ long and $6 \mathrm{~mm}$ wide, and the ring thickness was $15 \mathrm{~mm}$ to accommodate the beam windows. The samples were designed to initiate the failure in the cross-member.

\subsection{Microstructural characterization with EBSD}

To perform the EBSD analyses, a JEOL 6500F Field Emission Gun-SEM ${ }^{2}$ equipped with an EDAX Apollo Silicon Drift Detector and Hikari EBSD camera were used. The electron gun was set to an accelerating voltage of $20 \mathrm{kV}$ and tip current of $4 \mathrm{nA}$ for collecting the EBSD data. Prior to conducting EBSD analysis, all specimens were given a final colloidal silica (0.04 micron) polish. The post-processing of the collected data was performed using the TSL OIM Analysis software and no additional data clean-ups were performed.

\footnotetext{
${ }^{2}$ JEOL USA, Inc. 11 Dearborn Road, Peabody, MA 01960
} 
The data were obtained from both the cross-members (top, middle and bottom locations as shown in Figure 2 with yellow squares; both $/ /$ and $\perp$ to the Build Direction, BD) and the ring sections (from the portion near the loading mounts, yellow square in Figure 3b, // to BD) of the specimens. Data collection was performed using a hexagonal grid with varying sampling areas (from $\sim 1840 \times 1820 \mu \mathrm{m}^{2}$ on the cross-member $\perp$ to $\mathrm{BD}$, to $\sim 3670 \times 3640 \mu \mathrm{m}^{2}$ on the crossmember and on the ring section // to BD) and step sizes (from $2 \mu \mathrm{m}$ on the cross-member $\perp$ to $\mathrm{BD}$, to 5-6.5 $\mu \mathrm{m}$ on the cross-member and on the ring section // to $\mathrm{BD}$ ).

\subsection{Synchrotron $x$-ray diffraction measurements}

Synchrotron x-ray diffraction (SXRD) measurements on the cross-members extracted from undeformed samples were performed at Beamline 11-ID-C, Advanced Photon Source (APS), Argonne National Laboratory, in an effort to obtain the as-built textures. The cross-member sections were placed between the incident beam and a Perkin-Elmer 2-D detector in transmission geometry [32] with a sample to detector distance of $1296 \mathrm{~mm}$. The employed wavelength was $0.11165 \AA$ combined with a beam size of $0.7 \mathrm{~mm}$ x $0.7 \mathrm{~mm}$ x $4 \mathrm{~mm}$ (sample thickness). By virtue of the high energy $\mathrm{x}$-rays, the incident beam penetrated through the whole sample thickness; thus, providing bulk-averaged information. Three locations (top, middle and bottom) along the build direction were sampled (see indicated with yellow squares on the cross-member

in Figure 2). The diffraction data was collected by mounting the sections on a rotary stage and rotating $\omega$ from $0^{\circ}$ to $180^{\circ}$ with a $30^{\circ}$ step size to obtain full pole coverage as depicted in Figure 2. The Debye-Scherrer rings were converted into diffraction patterns using the Fit2D software with $10^{\circ}$ 'caking'. The Material Analysis using Diffraction (MAUD) software [33] was used to perform the Rietveld Refinements using the E-WIMV algorithm for the texture analysis. The raw 
pole figure data were processed further using the MTEX quantitative texture analysis software [34] for texture rotations and plotting the Pole Figures (PF).

\subsection{In-situ neutron diffraction measurements}

The in-situ lattice strain evolutions of the cross-members were measured using neutron diffraction at the VULCAN Beamline [35], Spallation Neutron Source (SNS), Oak Ridge National Laboratory (ORNL). The measurements were conducted in high-intensity (HI) mode that offers a neutron flux of up to $6.7 \times 10^{7} \mathrm{n} / \mathrm{s} / \mathrm{cm}^{2}$ to achieve good count statistics in a reasonable

amount of time, and a $2 \times 2 \times 2 \mathrm{~mm}^{3}$ gauge volume was used to ensure full burial of the neutron beam inside the sample.

For these measurements, the theta-shaped specimens were situated such that the cross-members were at a $45^{\circ}$ angle with respect to the incident neutron beam, Figure 3 . With this setup the axial and transverse components of strain were simultaneously detected making use of the two detectors (west and east banks) positioned at $\pm 90^{\circ}$ to the incident beam. The incident and diffracted beams were allowed to enter and exit through the as-built beam-windows with minimum attenuation.

The samples were loaded by applying compression on the mounting flats of the ring (indicated by the red arrows in Figure 3) which were translated into tensile stresses along the cross-member [30]. Initially the samples were held in place with a compressive pre-stress of $0.4 \mathrm{kN}$ cumulative load. This translated into a $\sim 7 \mathrm{MPa}$ tensile stress on the cross-member [30] and was taken as the "reference state" when calculating the lattice strains. The in-situ deformation was then carried 
out under load-control for a total of 15 loads up to maximum cumulative load levels (i.e., total loads experienced by the whole structure) of 66 and $58 \mathrm{kN}$ for the spot and line builds, respectively; followed by an unload in 4 steps back to $0.4 \mathrm{kN}$. During the neutron diffraction measurements the load was relaxed by $500 \mathrm{~N}$ from the peak applied load, at each studied level, in order to prevent further load relaxation during the measurements. Following this approach, no significant load relaxation was observed during the measurements.

The applied cumulative loads were selected for each theta sample (i.e., spot and line builds) to induce similar macroscopic engineering strains on their respective cross-members. For this, the 'cumulative load'-'strain on the cross-member' relations were used, which were obtained from ex-situ mechanical testing of sister samples of both builds. Accordingly, the highest loads (66 and $58 \mathrm{kN}$ for the spot and line builds, respectively) applied during the in-situ neutron diffraction measurements corresponded to $3.5 \%$ of macroscopic engineering strain on the cross-members [30]. In this context, the strains on the cross-members were incremented from the reference state ( 0\% strain) in $0.1 \%$ steps up to $1 \%$ strain followed by $0.5 \%$ increments up to $3.5 \%$ strain. For each load level, five points were mapped along the cross-members, i.e., Center, \pm 2 and $\pm 4 \mathrm{~mm}$ from the center, at 8 minutes per location (Figure $3 b$ ). The data reduction and single peak fittings were performed using the VULCAN Data Reduction and Interactive Visualization Software (VDRIVE) [36]. 


\section{Results}

\subsection{Build microstructures}

EBSD grain orientation and grain aspect ratio maps from both builds (cross-members and ring sections) are presented in Figures 4 and 5, respectively. Since qualitatively similar microstructures were observed along the crossmember (top, middle and bottom, Figure 2), only the results from the middle points (as representative) are presented in Figures 4 and 5 for brevity. In Figure 4 the color coding corresponds to different grain orientations per the inverse pole figure legend presented on the bottom left corner. On the other hand in Figure 5, the warmer colors correspond to higher level of equiaxed morphology with red being the most equiaxed and blue being the most columnar.

The micrographs of the cross-members (Figures 4 and 5) from along the build direction reveals that both the spot and the line builds are characterized with a columnar grain structure aligned largely with the build direction. Large columnar grains extending $>1 \mathrm{~mm}$ in length along the build direction are common in the spot build; based on measurements on the micrographs using ImageJ software [37]. It is also noteworthy to state that the microstructure is non-uniform with regions of very fine equiaxed stray grains; formed in pockets between the columnar grains. On the other hand, an overall qualitatively finer microstructure is observed in the line build showing regions of grains with 'closer to equiaxed' morphology compared to the spot build. Such microstructures are found to remain consistent as a function of height along the cross-member in each of the build strategies. 
However, perpendicular to the build direction a much finer microstructure is observed for both builds with more 'close to equiaxed' grains (Figure 5). Also, the grain orientations reveal the prominent presence of a preferred alignment of $\{200\}$ planes with their plane normals parallel to the build direction (Figure 4). The presence of such strong preferred orientations in both builds will be further presented in Section 3.2.

Finally, the ring sections also show the presence of columnar grains aligned with the build direction, consistent with the micrographs from the cross-members. A relatively coarser morphology with grains again extending $>1 \mathrm{~mm}$ is observed in the spot build (based on measurements with ImageJ [37]) compared to the line build in which the columnar structure is more broken up resulting in a finer microstructure with more 'closer to equiaxed' grains to form.

With columnar grains along the build direction, standard EBSD analysis methods cannot be used to reasonably deduce the grain sizes. Nevertheless, such information can be obtained from the cross-members perpendicular to the build direction where the grains show 'close to equiaxed' morphology. However, it must also be born in mind that the grain sizes obtained from perpendicular to the build direction correspond to the 'diameters' of the columnar grains.

The grain size (diameter) distributions obtained from the cross-members of both builds, perpendicular to the build direction, are presented in Figure 6. According to the results presented in Figure 6 both builds show a similar grain size distribution (perpendicular to the build direction) with the average values of $26.79 \mu \mathrm{m}$ and $30.28 \mu \mathrm{m}$ for the spot and line builds, respectively. 
Finally, in order to quantify the microstructures along the build direction, the lengths of the columnar grains are obtained with the ImageJ software [37]. The sampling was performed on all the micrographs using between 421 to 543 grains (including the stray grains), each. Accordingly, the average lengths of the columnar grains along the build direction are measured to be: $460 \mu \mathrm{m}$ and $305 \mu \mathrm{m}$ on the cross-members of the spot and line builds; and $331 \mu \mathrm{m}$ and $218 \mu \mathrm{m}$ on the ring sections of the spot and line builds. In agreement with the qualitative observations, these results show that an overall finer microstructure is obtained with the line build compared to the spot build. Further discussion on this subject will be presented in Section 4.1.

\subsection{Textures within the cross-members}

The textures from the top, middle and bottom sections (Figure 2) of the cross-member, obtained through SXRD, were observed to be qualitatively similar. Therefore, in order to save space, representative textural information from the center of the cross-members, i.e., middle location, are presented using the (111), (200), (220), (311), (331) and (420) PFs in Figure 7a and 7b for the spot and line builds, respectively. In Figure 7, the centers of the pole figures correspond to the build direction, perpendicular to the plane of the paper. Figure 7 shows that both samples are characterized with the presence of a strong (200) texture along the build direction (BD) and at $90^{\circ}$ to the BD. Further, the (111) poles are observed to be $\sim 55^{\circ}$ from the BD, and the (220) poles are situated at $\sim 45^{\circ}$ and at $90^{\circ}$ to the BD. The (311) poles are at $\sim 25^{\circ}$ and $72.5^{\circ},(331)$ at $\sim 46^{\circ}$ and $\sim 76^{\circ}$, and $(420)$ at $\sim 26^{\circ}, \sim 65^{\circ}$ and $90^{\circ}$ to the BD based on maximum intensities on the rings. 
This texture is observed to be approaching that of a single crystal. To investigate this further, the inter-planar angles, $\phi$, between the planes $\left(h_{1} k_{1} l_{1}\right)$ and $\left(h_{2} k_{2} l_{2}\right)$ were calculated for a cubic crystal through Eq. (1) [38]:

$$
\phi=\cos ^{-1}\left(\frac{h_{1} h_{2}+k_{1} k_{2}+l_{1} l_{2}}{\sqrt{\left(h_{1}^{2}+k_{1}^{2}+l_{1}^{2}\right)\left(h_{2}^{2}+k_{2}^{2}+l_{2}^{2}\right)}}\right)
$$

Following Eq. (1), the experimentally determined angles of various poles with respect to the build direction (presented above) are observed to match the interplanar angles predicted between $\left\{h_{1} k_{1} l_{1}\right\}=\{200\}$ and an $\left\{h_{2} k_{2} l_{2}\right\}$ such as $\{111\},\{220\}$, etc. Such an agreement suggests that the orientation of the observed texture poles are dominated by the strong $\{200\}$ fiber/"pseudo single crystal" texture along the build direction.

Finally, even though both samples reveal qualitatively similar textures, quantitative differences are observed suggesting a stronger (200) type texture in the spot build relative to the line build. This is evident when the relative maximums of the PFs, e.g., (200) vs. (111), are compared; revealing ratios of $25 / 5$ for the spot build and $8 / 3.1$ for the line build. Further investigation of this subject through the analysis of neutron diffraction profiles will be presented in Section 3.3. Additionally, the presence of stronger textures in the spot build relative to the line build can also be visualized by the sharper poles in the spot build as opposed to the more diffuse textures observed in the line build.

\subsection{In-situ loading with neutron diffraction}

The diffraction patterns obtained from the cross-members during in-situ neutron diffraction measurements are presented in Figure $8 \mathrm{a}$ and $8 \mathrm{~b}$ for the spot and the line builds, respectively; 
covering both the axial/build and transverse directions. In Figure 8 each run number corresponds to a different pattern, i.e., different load, along a given sample direction, i.e., axial/build or transverse. Also, each pattern corresponds to the ones obtained from the centers of the crossmembers (see Figure 3b).

In agreement with the PFs presented in Figure 7, a prominent presence of the (200) reflection is observed along the axial/build and transverse directions in both builds. Additionally, a strong presence of the (220) reflection along the transverse direction of both builds can be seen, again consistent with the bulk texture measurements from SXRD. Additionally, when the relative intensities of individual reflections, e.g., (311) vs (200), are compared between the builds, a stronger presence of (200) along the axial/build direction is observed in the spot build, compared to the line build, which is consistent with the PFs presented in Figure 7.

The intensity spikes observed in the first patterns (at $0.4 \mathrm{kN}$ applied cumulative load) are due to the longer count times at this load level, implemented to obtain improved statistics for the reference patterns. Finally, during these measurements only the peaks belonging to the fcc $\gamma$ matrix were detected.

\subsection{In-situ lattice strains}

The lattice strains, $\varepsilon_{\mathrm{hkl}}$, were calculated using Eq. (2) [39] from the d-spacings, $\mathrm{d}_{\mathrm{hkl}}$, measured insitu with neutron diffraction:

$$
\varepsilon_{h k l}=\frac{d^{h k l}-d_{0}^{h k l}}{d_{0}^{h k l}}
$$


where $\mathrm{d}_{0}^{\mathrm{hkl}}$ is the reference interplanar spacing. In this work, the strains are calculated with respect to the interplanar spacings of the $0.4 \mathrm{kN}$ holding load; i.e., the $\mathrm{d}_{\mathrm{hkl}}^{0}$ value corresponded to the d-spacing at the holding load. The changes in d-spacing of individual hkls as the samples are loaded/unloaded are visualized using 2D diffraction plots presented in Figure 9 for both builds and sample directions (i.e., axial and transverse). As the load levels are increased, an increase in the d-spacing values along the axial direction (accordingly decrease along the transverse direction due to the Poisson effect) can be observed for both builds followed by a decrease as the samples are unloaded (vice versa along the transverse direction). The evolution of the (111), (200), (220), (311) and (420) lattice strains are presented in Figure 10a and b as a function of the applied cumulative loads for the spot and the line builds, respectively; covering the axial and transverse directions. Following in Figure 10c and $\mathrm{d}$ are presented the evolution of the axial lattice strains as a function of the applied macroscopic strains on the cross-members, obtained from DIC [30], for the spot and line builds, respectively. In these figures the data points correspond to the average response of the five mapped locations on the cross-members (Center, \pm 2 and $\pm 4 \mathrm{~mm}$, Figure $3 \mathrm{~b}$ ) and the error bars to their standard deviation.

The relative changes in $\mathrm{d}$-spacing values $(\Delta \mathrm{d})$ differ for each reflection for the same change in load, pointing to elastic anisotropy. This highly anisotropic micro-mechanical behavior is observed in both builds, revealed by the large variation between the lattice strain responses of different hkl planes, consistent with the high elastic anisotropy of Ni-alloys [40]. Within this context, the (111) and (200) reflections mark the two extremes being the elastically stiffest and the most compliant planes, respectively [41]. 
Neutron diffraction measures the peak positions of the individual planes; and their relative shifts with respect to a reference value reveals information about the elastic strains of the atomic lattice. By the same token, diffraction cannot accurately measure the plastic strains as slipinduced deformation changes the relationship between applied load and the peak position/interplanar spacing, rendering it presently obfuscated. Accordingly, the development of lattice strains becomes non-linear once the grains start yielding since yielding would decelerate elastic strain increment [28, 42].

Figure 10c and 10d show that the onset of plasticity occurs first for the (111) and (220) reflections in both builds. More explicitly, the (111) and (220) planes both yield at an applied macrostrain of $\sim 0.5 \%$ in the spot build; whereas in the line build, yielding of (111) and (220) planes occurs $\sim 0.5 \%$ and $\sim 0.6 \%$ applied macrostrains, respectively. For (311) and (420), on the other hand, yield initiates between 0.6 and $0.7 \%$ applied macrostrains for both builds, respectively. Lastly for the (200) grains, the shift to plastic deformation occurs between $0.8 \%$ to $1 \%$ applied macroscopic strains for both builds. The transverse lattice strains show a similar distribution to those observed in the axial direction with $\varepsilon_{200}>\varepsilon_{311}>\varepsilon_{111}$ except for $\varepsilon_{220}$ and $\varepsilon_{420}$ which show an almost perfect overlap with $\varepsilon_{200}$ for both builds. This will be further discussed in Section 4.3.

Finally, the intergranular strains after unloading are expected to be in tensile character for the compliant reflections like the (200) and in compressive character for the stiffer ones like the (111). However, after unloading the theta specimens all of the (hkl) grain families showed compressive residual strains along the axial direction and tensile residual strains along the 
transverse direction (not shown here for the sake of brevity), implying that the ring sections have put the cross-members under compression upon unloading.

\section{Discussion}

\subsection{Relation between the scan strategies/build parameters and the resultant microstructures}

It was presented in Figures 4 and 5 that both the spot and the line builds revealed a highly oriented and 'close to equiaxed' grain structure perpendicular to the build direction with a similar grain size distribution as shown in Figure 6. On the other hand, the most distinct differences between the builds were observed along the build direction. As shown in Figures 4 and 5, spot and line builds both resulted in columnar structures along the build direction, with the line build exhibiting a smaller grain size with more 'closer to equiaxed' grains compared to the spot build, based on both qualitative and quantitative observations.

Overall, the respective grain sizes and morphologies in the spot and line builds can be related to the respective beam scan strategies and parameters used in the context of heat transfer conditions during solidification.

In classical nucleation and growth, the initial melt and rapid cooling causes massive nucleation and a random microstructure. However, once grains are established at the bottom of the melt pool, the rapid cooling promotes the atoms to lock onto the seed grains with the preferred growth directions and favorable thermodynamics take over. Similar to welding, the variables that control grain formation upon solidification in AM are the growth rate (R), temperature gradient $(\mathrm{G})$, and 
the undercooling in the melt. Due to the nature of the EBM process and AM processes in general, the high thermal gradients (upwards of $10^{3} \mathrm{~K} / \mathrm{m}$ ) and high solidification velocities are ideal for promoting the growth of columnar grains aligned with the build direction [43, 44]. As has been demonstrated by Dehoff et al. [22] and Dinda et al. [45], the grain structure can be influenced through manipulations of the heat source and scan strategy resulting in manipulation of both the thermal gradient and undercooling during solidification.

For the spot build strategy, the addition of a new layer results in the formation of a localized shallow and narrow melt pool that largely promote the continuation of already existing grains over the nucleation of new grains. On the other hand, in the case of the line build strategy, the addition of a new layer results in the meltback of 3-4 layers of the already deposited material, netting a total melt pool depth of 200-250 $\mu \mathrm{m}$ [46]. As a result, a large undercooling develops in the melt pool leading to an increase in the rate of nucleation of new grains $[47,48]$ that span the re-melted material and aligned with the near vertical thermal gradient in the melt pool.

Overall, this study is indicative of the effect of build parameters on influencing the resultant microstructures. Thus, further research on this front proves critical as it holds great potential for future uptake of AM.

\subsection{Axial lattice strains and load partitioning}

In Section 3.4., the (111) and the (220) orientations were shown to reach the yield point earlier than the (311), (420) and the (200) orientations, despite being elastically stiffer. Such phenomenon occurs due to the stiffer orientations bearing a higher portion of the applied load 
and "shielding" the more compliant ones like the $(200)$ [42, 49]. Holden et al. [42] uses the following analogy to help explain this behavior by assuming a polycrystalline material that is formed of a set of parallel columns of single fiber grains with different orientations (e.g., see Figure 11). Considering the fact that the (200) orientation has the lowest Taylor Factor, one could assume that it would yield at the lowest stress compared to the other orientations. However, when this grain is loaded along with the other columnar grains, the stiffer grains would reduce the load on the (200) grains to keep the strains in all the grains equivalent per the isostrain condition. Therefore, the linear strain required to reach the yield stress would not be reached in the (200) grains until the stiffer grains have yielded first.

Here, both builds show the dominant presence of the $\{200\}$ orientation along the build direction, almost like a 200 single crystal; as revealed by Figures 4,7 and 8 . Thus, with the virtually negligible fraction of the stiffer (111) and (220) orientations along the build/axial direction, the shielding effect is not very prominent. Evidently, these stiffer orientations yield at very early levels of applied macroscopic strains $(\sim 0.5 \%)$ accompanied with an elastic saturation and shifting into total plasticity indicated by the flat-lining behavior in Figure 10c and d.

The scarcity of the elastically stiffer orientations is also expected to result in a lower bulk elastic modulus compared to more randomly oriented counterparts. According to the FEM analysis reported in our recent work [30], bulk elastic moduli of 162 and $168 \mathrm{GPa}$ were achieved for spot and line builds, respectively; compared to the bulk elastic modulus of $200 \mathrm{GPa}$ reported for conventionally manufactured IN718 [50]. For a Waspaloy (a nickel-base superalloy) Stone et al. has reported the Diffraction Elastic Modulus of the (200) orientation, $\mathrm{E}_{200}$, as $171.8 \mathrm{GPa}$ [51] 
whereas Repper et al. reported to observe $\mathrm{E}_{200}=158-166 \mathrm{GPa}$ for an IN718 [52]. Accordingly, considering the strong presence of (200) in both builds it is plausible that the bulk elastic moduli will be closer to that of (200). Furthermore, the slightly lower modulus of spot build is also in agreement with the stronger presence of (200) compared to line build.

With the initiation of plasticity in the stiffer grains, the portion of the elastic load they (e.g., 111 and 220) would have carried will be transferred to the remainder of the grains that have not yet yielded. This will lead to the grains with orientations to the applied load that have not yet yielded (e.g., 200) to be increasingly strained elastically per given load increment while the plastically deforming grains remain at a stress corresponding to the critical resolved shear stress, unable to sustain elastic strains [28, 42, 51].

In order to better visualize these strain redistributions, the lattice strains of the different grain families can be evaluated as a function of the stresses experienced by the cross-members. However, due to the rather complex sample geometry, it was not possible to directly deduce the macroscopic tensile stresses on the cross-members. Instead, the stresses sustained by the crossmembers, $\sigma_{\mathrm{ij}}$, are inferred from the lattice strains using Eq. (3) [39];

$$
\sigma_{i j}=\frac{E_{h k l}}{\left(1+v_{h k l}\right)}\left[\varepsilon_{i j}+\frac{v_{h k l}}{\left(1-2 v_{h k l}\right)}\left(\varepsilon_{11}^{h k l}+\varepsilon_{22}^{h k l}+\varepsilon_{33}^{h k l}\right)\right]
$$

where $E_{\mathrm{hkl}}$ is the Young's Modulus, $v_{\mathrm{hkl}}$ is the Poisson's Ratio and $\varepsilon_{\mathrm{ij}}$ is the measured lattice strain in a given sample direction. Here, $\varepsilon_{\mathrm{ii}}$ are the principal strains. For the nomenclature of the current work, $\varepsilon_{11}$ is taken as the loading/build/axial direction, $\varepsilon_{22}$ as the normal and $\varepsilon_{33}$ as the transverse directions. Furthermore $\varepsilon_{22}$ was regarded as equal to $\varepsilon_{33}$, per tensile deformation symmetry. This is an appropriate assumption as the loading is applied in known directions 
coincident with the sample geometry defined, and the stresses calculated are defined as only the stresses from an unloaded state. For the determination of stresses, $v$ was taken as 0.3 whereas $\mathrm{E}$ values of 162 and $168 \mathrm{GPa}$ [30] were used for spot and line builds, respectively.

The (311) reflection (i.e., $\varepsilon^{311}$ ) was selected for the calculation of stresses per Eq. (3), as it is reported to be weakly affected by the intergranular strains for an FCC material [39]. The resultant stress-strain plots are presented in Figure 12a and $\mathrm{b}$ for spot and line builds respectively, covering both the axial and transverse directions. It should, however, be born in mind that by following this approach the (311) grains are always going to show a linear elastic stress-strain behavior, and the response of the remaining grain families will be relative to that of the (311).

Nevertheless, the strain redistributions can still be deduced from these plots. The load partitioning is manifested in a slope change in the observed stress-lattice strain response, and it is particularly prominent for the (200) reflection. The response of the (200) grains is fitted with two lines corresponding to two different slopes. The slope decreases (i.e., the level of elastic strain per stress increment increases) as the stiffer (111) and (220) orientations start to yield and part of the elastic load they would have carried is transferred to the (200) oriented grains. This is followed by the yield of (200) which corresponds to the dip at the end of the decreased slope. Overall, similar responses are observed in both builds in terms of load partitioning mechanisms.

One can also observe the difference in the maximum (311) axial stresses between the two builds in Figure 12. However, this figure is primarily intended to illustrate the load partitioning between 
different (hkl)s, and the (311) stresses obtained with Eq. (3) (following the reasoning presented earlier) are strictly related to the lattice strain responses of the (311) orientation in both samples. Thus, the observed maximum stress difference between the two builds is a direct consequence of the difference in the lattice strain evolution of the (311) planes.

It was shown in Figure 8 that the line build was characterized with a relatively stronger presence of (311) planes in both axial and transverse directions compared to spot build. This stronger presence of the elastically stiffer (311), e.g., compared to (200), in the line build could lead to the accumulation of higher elastic strains, $\varepsilon_{311}$. In fact, such behavior can be seen in Figure 10. When comparing the axial lattice strain responses of the spot and line builds (Figure 10c and d) it can be observed that the (311) lattice strains continue increasing in an elastic character in the line build whereas it almost stagnates in the spot build, past yielding. This response, when coupled with the slightly higher elastic modulus of the line build, can help explain the observed difference in the maximum (311) axial stresses. Nevertheless, the relatively higher presence of the elastically stiffer orientations in the line build could still indicate a stiffer cross member along the build direction compared to the spot build.

\subsection{Effects of texture on the transverse strain response}

It was presented in Section 3.4. that the transverse lattice strain evolutions of the (220) and (420) reflections closely overlap with that of the (200) reflection. This behavior posed rather irregular considering, for instance, the more typical evolution of the (220) transverse lattice strains as a function of applied load, which is reported to be between those of the (111) and (311) reflections, 
and above that of the $(200)[28,29,53]$. To investigate this issue, the hkl-specific Poisson's ratios, $v_{\mathrm{hkl}}$, are calculated from the in-situ deformation data following Eq. (4):

$$
v_{\mathrm{hkl}}=-\frac{\varepsilon_{\mathrm{tr}}^{\mathrm{hk} 1}}{\varepsilon_{\mathrm{ax}}^{\mathrm{hkl}}}
$$

where $\varepsilon_{\mathrm{ax}}$ and $\varepsilon_{\mathrm{tr}}$ are the axial and transverse lattice strains, respectively. The experimentally determined $v_{\mathrm{hkl}}$ values, following Eq. (4), for the (200), (220), (311) and (420) orientations are presented in Table 1 for both builds. $v_{111}$ values were not calculated due to the high level of scatter in the data resulting from the very weak presence of (111) in both the axial and the transverse directions. For comparison, $v_{\mathrm{hkl}}$ values determined using the Kröner model (for pure $\mathrm{Ni}$, assuming random orientation) from Ref. [39] are also presented in Table 1. Following the data presented in Table 1, a comparison between the experimental and model [39] results reveal that while the $v_{200}$ and the $v_{311}$ values agree within acceptable tolerance, large differences are observed for the $v_{220}$ and the $v_{420}$ values, with the biggest difference being observed for the (220) orientation.

To illustrate this irregularity better, the expected transverse lattice strain evolutions of (220) and (420) reflections are calculated from the axial lattice strains (see Figure 12c and d) following Eq. (4) and using $v_{\mathrm{hkl}}$ values from the Kröner model (see Table 1) [39]. These calculated values of tranverse strain (half-full symbols, emphasized with a larger font) are presented together with the experimentally measured ones (empty symbols) in Figure 12c and 12d for spot and line builds, respectively. Even though such calculated results may not be very accurate after the onset of plastic deformation, the deviation from the experimentally measured results is still well illustrated during elastic deformation. 
Texture can have a significant effect on the lattice strain measurements compared to more randomly oriented materials, and during tensile deformation, the effect of grain orientations on the observed strain response can vary significantly between the loading and transverse directions. As explained in $[29,54]$, the strain response of a family of grains with their (hkl) plane normals aligned along the loading direction will not be greatly affected by a rotation around [hkl] which will only alter the planes that are transverse to the direction of the load. On the contrary, for a family of grains whose (hkl) plane normals are aligned parallel to the transverse direction, a rotation around [hkl] can bring various planes to align their normals along the loading direction, i.e., can change the orientation relative to the tensile axis and thus significantly alter the transverse strain response. For instance, a family of grains whose (hkl) plane normals are aligned parallel to the transverse direction could have two individual grains: one aligned with its (111) plane normal along the loading direction, i.e, the stiffest, and the other its (200) plane normal, i.e., the most compliant. Accordingly, the observed transverse strain response is expected to be an average of all the grains aligned with their (hkl) plane normals parallel to the transverse direction however at different orientations with respect to the tensile axis; within the sampled volume by neutrons.

As revealed by the S-XRD and EBSD results, both builds are characterized with strong (200) textures along their build/loading directions which can then significantly affect the transverse strain response per the reasoning presented above. It is plausible that the vast majority of the (hkl) planes situated to diffract in the transverse direction will have one of their <200> orientations aligned with the loading direction. Accordingly for a given $\left\{h_{1} k_{1} l_{1}\right\}$ grain family whose plane normals are parallel to the transverse direction, it is highly likely to observe a 
pseudo-single crystal-like response where all of the grains within the studied volume are strained along the same direction; instead of various grains of this family having different [hkl] aligned along the loading direction and presenting an average response of all these variations.

The presence of such distinct and strong textures, nevertheless, makes this an interesting casestudy where the loading direction of a transverse orientation can be estimated fairly easily compared to more randomly oriented materials. For instance, focusing on the angular relationship between the $\{200\},\{220\}$ and $\{420\}$ grain families in the transverse direction and the $\{200\}$ grain family in the axial/loading direction, one can find that the interplanar angle between certain members of these grain families equals to $90^{\circ}$ per Eq. (1). Such pairs include some of the following: $(200)_{\mathrm{ax}}-(022)_{\mathrm{tr}},(200)_{\mathrm{ax}}-(024)_{\mathrm{tr}},(200)_{\mathrm{ax}}-(002)_{\mathrm{tr}},(002)_{\mathrm{ax}}-(220)_{\mathrm{tr}},(002)_{\mathrm{ax}}-$ $(420)_{\operatorname{tr}}$ and $(002)_{\mathrm{ax}}-(200)_{\mathrm{tr}}$. This is also consistent with the texture results presented in Figure $7 \mathrm{a}$ and $b$ where the distribution of the texture fibers fits such a relation. Based on this angular relationship, the Poisson contraction of such transverse grain families can be estimated for a fixed loading direction, i.e., pulled along a $<002>$ direction.

Wojciechowski [55] has come up with the following expressions for a cubic material to calculate the Poisson's ratio for a given loading direction at an angle $\chi$ with respect to the z-axis (001) whose projection on the $\mathrm{x}-\mathrm{y}$ plane makes an angle $\varphi$ with the $\mathrm{x}$-axis (100) (see Figure 13); using the three independent compliance constants $S_{11}, S_{12}$ and $S_{44}$ :

$$
v=-\frac{A \frac{S_{12}}{S_{11}}+B\left[\frac{S_{44}}{S_{11}}-2\right]}{16\left[C+D\left(2 \frac{S_{12}}{S_{11}}+\frac{S_{44}}{S_{11}}\right)\right]}
$$


Where,

$$
\begin{gathered}
\mathrm{A}=2\left(53+4 \cos (2 \chi)+7 \cos (4 \chi)+8 \cos (4 \varphi) \sin ^{4} \chi\right) \\
\mathrm{B}=-11+4 \cos (2 \chi)+7 \cos (4 \chi)+8 \cos (4 \varphi) \sin ^{4} \chi \\
\mathrm{C}=8 \cos ^{4} \chi+6 \sin ^{4} \chi+2 \cos (4 \varphi) \sin ^{4} \chi \\
\mathrm{D}=2\left(\sin ^{2}(2 \chi)+\sin ^{4} \chi+\sin ^{2}(2 \varphi)\right)
\end{gathered}
$$

Following Eq.s (5) and (6) the Poisson's ratios can be calculated, e.g., for three different grains all of which are loaded along a [002] direction, but contracting distinctly along [200], [220] and [420] directions, respectively. Using the elastic constants from Daymond et al. [40], for a nickelbase superalloy, the Poisson's ratio is then found to be 0.39 for the [220] and 0.4 for the [200], and the [420] directions. This goes to reveal that the Poisson contraction is expected to be almost the same for all the planes that are at a $90^{\circ}$ angle (i.e., perpendicular) to a fixed loading direction, regardless of their orientation. Indeed, these findings are in agreement with the experimentally determined evolution of the transverse lattice strains.

Overall, both the axial and transverse lattice strain evolutions show the influence of strong build textures on the observed deformation response; with the effects being more pronounced in the case of transverse lattice strains. The findings of this work further emphasize the importance of texture control, via build parameter optimization, and its influence on potential design of microstructures and micromechanical properties.

\section{Summary and Conclusions}

In this work, complex theta-shaped specimens were additively manufactured from Inconel 718 powders using the electron beam melting technique and employing two sets of build strategies. 
The samples were characterized using the electron back scatter, synchrotron x-ray and in-situ neutron diffraction techniques. The conclusions are as follows:

1. The build strategies are found to greatly affect the grain morphologies. Even though both builds were characterized with 'close to equiaxed' grains perpendicular to the build direction, columnar grains were observed along the build direction for both builds. Rather large grains with lengths $>1 \mathrm{~mm}$ along the build direction (based on measurements on the micrographs using the ImageJ software) were observed for the spot build whereas a finer microstructure was observed for the line build. The finer grain structure observed in the line build was related to the presence of a melt-back effect where the electron beam remelted the previously deposited layers (3-4 layers) at each step causing a large undercooling to develop that lead to an increase in the nucleation of new grains. On the other hand, the rather localized shallow melt pool employed in the spot build strongly promoted an epitaxial growth between the build layers effectively resulting in large columnar grains that extend several build layers.

2. Both builds are characterized with strong (200) textures along the build direction and at $90^{\circ}$ to it; i.e., also spanning the transverse and normal directions. However, the textures in general, are observed to be stronger/sharper in the spot build and relatively weaker/more diffuse in line build. The presence of a strong preferred (200) orientation along the build and transverse directions was also confirmed by the EBSD grain orientation maps and the neutron diffraction profiles. Furthermore, the relatively weaker/more diffuse texture of line build compared to the spot build was also visible in the neutron diffraction profiles. 
3. The evolution of axial lattice strains revealed a highly anisotropic behavior in both builds with the elastically stiffer (111) and (220) orientations yielding earlier than the more elastically compliant ones like (200). The low presence of the elastically stiffer orientations prohibited an effective shielding of the more compliant orientations; and with the early yielding of the stiffer orientations part of the elastic load they would have carried is transferred to the compliant ones like (200). Furthermore, the strong presence of the compliant (200) orientation agreed with the relatively low bulk moduli of both builds compared to the conventionally manufactured Inconel 718.

4. The evolution of the transverse lattice strains in both builds showed an irregular behavior with $\{200\},\{220\}$ and $\{420\}$ orientations showing an almost perfect overlap. This was related to the presence of heavy almost single crystal-like build textures which led to these transverse planes all being loaded along $\{002\}$ thus resulting in almost the same Poisson contraction.

Overall the theta-shaped specimens, as model complex load bearing structures, were successfully manufactured with both build strategies while the different strategies resulted in different grain morphologies and texture intensities. Furthermore, the textures are observed to affect the micromechanical behavior in both builds and have to be optimized in future studies. 


\section{Acknowledgements}

This research was sponsored by the Laboratory Directed Research and Development Program of Oak Ridge National Laboratory, managed by UT-Battelle, LLC, for the U. S. Department of Energy. Research at MDF was sponsored by the U.S. Department of Energy, Office of Energy Efficiency and Renewable Energy, Advanced Manufacturing Office, under contract DE-AC0500OR22725 with UT-Battelle, LLC. The research at ORNL's Spallation Neutron Source was sponsored by the Scientific User Facilities Division, Office of Basic Energy Sciences, US Department of Energy. This research used resources of the Advanced Photon Source, a U.S. Department of Energy (DOE) Office of Science User Facility operated for the DOE Office of Science by Argonne National Laboratory under Contract No. DE-AC02-06CH11357. EC and TRW would like to thank Mr. Tom Geer for metallographic sample preparation. EC would also like to acknowledge Dr. Olaf J. Borkiewicz for his helps during the measurements at APS. 


\section{References}

[1] C.A. Brice, W.H. Hofmeister. Determination of bulk residual stresses in electron beam additive-manufactured aluminum, Metall Mater Trans A 44A (2013) 5147-5153.

[2] R.R. Dehoff, S.S. Babu. Characterization of interfacial microstructures in 3003 aluminum alloy blocks fabricated by ultrasonic additive manufacturing, Acta Mater 58 (2010) 4305-4315. [3] W.J. Sames, K.A. Unocic, R.R. Dehoff, T. Lolla, S.S. Babu. Thermal effects on microstructural heterogeneity of Inconel 718 materials fabricated by electron beam melting, J Mater Res 29 (2014) 1920-1930.

[4] L.E. Murr, E. Martinez, S.M. Gaytan, D.A. Ramirez, B.I. Machado, P.W. Shindo, J.L. Martinez, F. Medina, J. Wooten, D. Ciscel, U. Ackelid, R.B. Wicker. Microstructural architecture, microstructures, and mechanical properties for a nickel-base superalloy fabricated by electron beam melting, Metall Mater Trans A 42A (2011) 3491-3508.

[5] L.E. Murr, E.V. Esquivel, S.A. Quinones, S.M. Gaytan, M.I. Lopez, E.Y. Martinez, F. Medina, D.H. Hernandez, E. Martinez, J.L. Martinez, S.W. Stafford, D.K. Brown, T. Hoppe, W. Meyers, U. Lindhe, R.B. Wicker. Microstructures and mechanical properties of electron beamrapid manufactured Ti-6Al-4V biomedical prototypes compared to wrought Ti-6Al-4V, Mater Charact 60 (2009) 96-105.

[6] A. Strondl, R. Fischer, G. Frommeyer, A. Schneider. Investigations of MX and gamma '/gamma" precipitates in the nickel-based superalloy 718 produced by electron beam melting, Mater Sci Eng A-Struct 480 (2008) 138-147.

[7] A. Strondl, M. Palm, J. Gnauk, G. Frommeyer. Microstructure and mechanical properties of nickel based superalloy IN718 produced by rapid prototyping with electron beam melting (EBM), Mater Sci Tech SER 27 (2011) 876-883. 
[8] R. Dehoff, C. Duty, W. Peter, Y. Yamamoto, W. Chen, C. Blue, C. Tallman. Case study: Additive manufacturing of aerospace brackets, Mater Sci Forum 171 (2013) 19-22.

[9] P.L. Blackwell. The mechanical and microstructural characteristics of laser-deposited IN718, J Mater Process Tech 170 (2005) 240-246.

[10] H. Qi, M. Azer, A. Ritter. Studies of standard heat treatment effects on microstructure and mechanical properties of laser net shape manufactured Inconel 718, Metall Mater Trans A 40A (2009) 2410-2422.

[11] Y. Tian, D. McAllister, H. Colijn, M. Mills, D. Farson, M. Nordin, S. Babu.

Rationalization of microstructure heterogeneity in Inconel 718 builds made by the direct laser additive manufacturing process, Metall Mater Trans A 45A (2014) 4470-4482.

[12] T. Vilaro, C. Colin, J.D. Bartout, L. Naze, M. Sennour. Microstructural and mechanical approaches of the selective laser melting process applied to a nickel-base superalloy, Mater Sci Eng A-Struct 534 (2012) 446-451.

[13] K.N. Amato, S.M. Gaytan, L.E. Murr, E. Martinez, P.W. Shindo, J. Hernandez, S. Collins, F. Medina. Microstructures and mechanical behavior of Inconel 718 fabricated by selective laser melting, Acta Mater 60 (2012) 2229-2239.

[14] L.M. Sochalski-Kolbus, E.A. Payzant, P.A. Cornwell, T.R. Watkins, S.S. Babu, R.R. Dehoff, M. Lorenz, O. Ovchinnikova, C. Duty. Comparison of residual stresses in Inconel 718 simple parts made by electron beam melting and direct laser metal sintering, Metall Mater Trans A 46A (2015) 1419-1432.

[15] S.S. Al-Bermani, M.L. Blackmore, W. Zhang, I. Todd. The origin of microstructural diversity, texture, and mechanical properties in electron beam melted Ti-6Al-4V, Metall Mater Trans A 41A (2010) 3422-3434. 
[16] S.A. David, J.M. Vitek, M. Rappaz, L.A. Boatner. Microstructure of stainless-steel single-crystal electron-beam welds, Metall Mater Trans A 21 (1990) 1753-1766.

[17] M. Rappaz, S.A. David, J.M. Vitek, L.A. Boatner. Development of microstructures in Fe15Ni-15Cr single-crystal electron-beam welds, Metall Trans A 20 (1989) 1125-1138.

[18] L.E. Murr, S.M. Gaytan, D.A. Ramirez, E. Martinez, J. Hernandez, K.N. Amato, P.W. Shindo, F.R. Medina, R.B. Wicker. Metal fabrication by additive manufacturing using laser and electron beam melting technologies, J Mater Sci Technol 28 (2012) 1-14.

[19] A. Strondl, S. Milenkovic, A. Schneider, U. Klement, G. Frommeyer. Effect of processing on microstructure and physical properties of three nickel-based superalloys with different hardening mechanisms, Adv Eng Mater 14 (2012) 427-438.

[20] R. Dehoff, M. Kirka, F. List III, K. Unocic, W. Sames. Crystallographic texture engineering through novel melt strategies via electron beam melting: Inconel 718, Mater Sci Tech SER 31 (2015) 939-944.

[21] C. Körner, H. Helmer, A. Bauereiß, R.F. Singer. Tailoring the grain structure of IN718 during selective electron beam melting. MATEC Web of Conferences, vol. 14: EDP Sciences, 2014. p.08001.

[22] R. Dehoff, M. Kirka, W. Sames, H. Bilheux, A. Tremsin, L. Lowe, S. Babu. Site specific control of crystallographic grain orientation through electron beam additive manufacturing, Mater Sci Tech SER 31 (2015) 931-938.

[23] K.N. Amato, J. Hernandez, L.E. Murr, E. Martinez, S.M. Gaytan, P.W. Shindo, S. Collins. Comparison of microstructures and properties for a Ni-base superalloy (alloy 625) fabricated by electron and laser beam melting, J Mater Sci Res 1 (2012) 3-41. 
[24] P. Rangaswamy, M.L. Griffith, M.B. Prime, T.M. Holden, R.B. Rogge, J.M. Edwards, R.J. Sebring. Residual stresses in LENS (R) components using neutron diffraction and contour method, Mater Sci Eng A-Struct 399 (2005) 72-83.

[25] A.J. Durelli, S. Morse, V. Parks. The theta specimen for determining tensile strength of brittle materials, Mater Res Standard 2 (1962) 114-117.

[26] G.D. Quinn. Fractographic analysis of very small theta specimens. in: Dusza J, Danzer R, Morrell R, Quinn GD, (Eds.). Key Eng Mat, vol. 409. 2009. pp. 201-208.

[27] K. An, B. Clausen, A.D. Stoica, B.L. Armstrong, H.D. Skorpenske, X.-L. Wang. In-situ neutron diffraction study of phase stress evolutions in a Ni-based porous anode solid oxide fuel cells under uniaxial load, Appl Phys A-Mater 99 (2010) 579-584.

[28] B. Clausen, T. Lorentzen, M.A.M. Bourke, M.R. Daymond. Lattice strain evolution during uniaxial tensile loading of stainless steel, Mater Sci Eng A-Struct 259 (1999) 17-24.

[29] M.R. Daymond, C.N. Tome, M.A.M. Bourke. Measured and predicted intergranular strains in textured austenitic steel, Acta Mater 48 (2000) 553-564.

[30] E. Cakmak, T.R. Watkins, J.R. Bunn, R.C. Cooper, P.A. Cornwell, Y. Wang, L.M. Sochalski-Kolbus, R.R. Dehoff, S.S. Babu. Mechanical characterization of an additively manufactured Inconel 718 theta shaped specimen, Metall and Mat Trans A 47 (2016) 971-980.

[31] M.M. Kirka. Personal Communication, 2015.

[32] E. Cakmak, H. Choo, K. An, Y. Ren. A synchrotron X-ray diffraction study on the phase transformation kinetics and texture evolution of a TRIP steel subjected to torsional loading, Acta Mater 60 (2012) 6703-6713. 
[33] L. Lutterotti, S. Matthies, H.-R. Wenk, A. Schultz, J. Richardson Jr. Combined texture and structure analysis of deformed limestone from time-of-flight neutron diffraction spectra, $\mathrm{J}$ Appl Phys 81 (1997) 594-600.

[34] F. Bachmann, R. Hielscher, H. Schaeben. Texture analysis with MTEX - free and open source software toolbox, Sol St Phen 160 (2010) 63-68.

[35] K. An, H.D. Skorpenske, A.D. Stoica, D. Ma, X.-L. Wang, E. Cakmak. First in situ lattice strains measurements under load at Vulcan, Metall Mater Trans A 42A (2011) 95-99.

[36] K. An. VDRIVE-Data reduction and interactive visualization software for event mode neutron diffraction. ORNL Report: Oak Ridge National Laboratory, 2012.

[37] C.A. Schneider, W.S. Rasband, K.W. Eliceiri. NIH image to ImageJ: 25 years of image analysis, Nat Methods 9 (2012) 671-675.

[38] B. Cullity, S. Stock. Elements of x-ray diffraction. Third Edition ed., Prentice Hall, 2001.

[39] M.T. Hutchings, P.J. Withers, T.M. Holden, T. Lorentzen. Introduction to the characterization of residual stress by neutron diffraction, Taylor and Francis, Boca Raton, FL, 2005.

[40] M.R. Daymond, M. Preuss, B. Clausen. Evidence of variation in slip mode in a polycrystalline nickel-base superalloy with change in temperature from neutron diffraction strain measurements, Acta Mater 55 (2007) 3089-3102.

[41] M.R. Daymond, M.A.M. Bourke, R.B. VonDreele, B. Clausen, T. Lorentzen. Use of Rietveld refinement for elastic macrostrain determination and for evaluation of plastic strain history from diffraction spectra, J Appl Phys 82 (1997) 1554-1562. 
[42] T.M. Holden, R.A. Holt, A.P. Clarke. Intergranular strains in Inconel-600 and the impact on interpreting stress fields in beat steam-generator tubing, Mater Sci Eng A-Struct 246 (1998) $180-198$.

[43] M. Gäumann, C. Bezencon, P. Canalis, W. Kurz. Single-crystal laser deposition of superalloys: processing-microstructure maps, Acta Mater 49 (2001) 1051-1062.

[44] W. Kurz, D.J. Fisher. Fundamentals of solidification. 3rd Edition ed., Trans Tech Publications, Switzerland, 1992.

[45] G. Dinda, A. Dasgupta, J. Mazumder. Texture control during laser deposition of nickelbased superalloy, Scripta Mater 67 (2012) 503-506.

[46] M.M. Kirka, K. Unocic, N. Raghavan, R.R. Dehoff, S.S. Babu. The macrostructure and microstructure development of Inconel 718 during the electron beam melting process. submitted to J Mater.

[47] J. Hunt. Steady state columnar and equiaxed growth of dendrites and eutectic, Mater Sci Eng 65 (1984) 75-83.

[48] N. Raghavan, S. Pannala, S.S. Babu, R.R. Dehoff, M.M. Kirka, S. Simonovic, J. Turner, N. Carlson. Numerical modeling of heat transfer and study of the influence of input parameters on tailoring the grain morphology of IN718 in electron beam additive manufacturing. submitted to Acta Mater.

[49] J. Coakley, D. Dye. Lattice strain evolution in a high volume fraction polycrystal nickel superalloy, Scripta Mater 67 (2012) 435-438.

[50] Technical Bulletin: "Inconel Alloy 718", Special Metals Corporation, 2007.

[51] H.J. Stone, T.M. Holden, R.C. Reed. On the generation of microstrains during the plastic deformation of Waspaloy, Acta Mater 47 (1999) 4435-4448. 
[52] J. Repper, P. Link, M. Hofmann, C. Krempaszky, W. Petry, E. Werner. Interphase microstress measurements in IN 718 by cold neutron diffraction, Appl Phys A-Mater 99 (2010) $565-569$.

[53] N. Jia, R.L. Peng, D.W. Brown, B. Clausen, Y.D. Wang. Tensile deformation behavior of duplex stainless steel studied by in-situ time-of-flight neutron diffraction, Metall Mater Trans A 39A (2008) 3134-3140.

[54] B. Clausen, T. Lorentzen, T. Leffers. Self-consistent modelling of the plastic deformation of fcc polycrystals and its implications for diffraction measurements of internal stresses, Acta Mater 46 (1998) 3087-3098.

[55] K.W. Wojciechowski. Poisson's ratio of anisotropic systems, Comp Met Sci Technol 11 (2005) 73-79. 


\section{$\underline{\text { Figure Captions }}$}

Figure 1. Schematics illustrating the build patterns of the a) Spot and b) Line build strategies. The Spot Build consists of melting individual 'spots' one point at a time in a pattern whereas the Line Build does a continuous melt following a snake raster pattern.

Figure 2. A schematic of the synchrotron x-ray diffraction measurement setup in transmission geometry. BD, TD, and ND refer to the build, transverse, and normal directions of the crossmember, respectively; and $\omega$ corresponds to the rotation axis for the texture measurements. Also shown on the schematic are the locations along the cross-member (see yellow squares) from which the data was obtained.

Figure 3. a) A schematic of the in-situ loading neutron diffraction measurement setup. The signal from the planes perpendicular (i.e., their plane normals parallel) to the build/axial direction and along the cross-member is received in the east bank of detectors. Likewise, the signal from the planes perpendicular to the transverse direction is received by the west bank of detectors. The red arrows correspond to the direction of the applied compressive load on the theta sample which places the cross-member in tension. Note the beam "windows" which allow the passage of the incident and diffracted beams to and from the region of interest with minimal attenuation; b) The location of the neutron diffraction mapping points along the cross-member. A.D., T.D., and N.D. refer to the axial, transverse and normal directions, respectively. Also shown here is the location on the ring section (see yellow square) from where the EBSD measurements were taken. 
Figure 4. EBSD grain orientation maps obtained from the cross-members parallel (//) and perpendicular $(\perp)$ to the build direction $(\mathrm{BD})$ and from the ring sections parallel $(/ /)$ to $\mathrm{BD}$; presented for both the spot and the line builds. In the case of //BD, the build direction lies along the vertical axis of the micrographs (see the arrow) whereas it is pointing out of the plane of the paper in the case of $\perp \mathrm{BD}$.

Figure 5. EBSD grain aspect ratio maps obtained from the cross-members parallel (//) and perpendicular $(\perp)$ to the build direction $(\mathrm{BD})$ and from the ring sections parallel $(/ /)$ to $\mathrm{BD}$; presented for both the spot and the line builds. In the case of //BD, the build direction lies along the vertical axis of the micrographs (see the arrow) whereas it is pointing out of the plane of the paper in the case of $\perp \mathrm{BD}$. The warmer colors correspond to more equiaxed grains with red being the most equiaxed and blue being the most columnar.

Figure 6. Grain size (Diameter) distributions obtained from the cross-members of both the spot and the line builds, perpendicular to the build direction (see Figures 4 and 5).

Figure 7. (111), (200), (220), (311), (331) and (420) pole figures for a) Spot Build and b) Line Build obtained with SXRD. The Build Direction is at the center of the pole figures pointing out of the plane of the paper. The darker colors represent higher levels of intensity, and the intensity levels are on linear scale.

Figure 8. Neutron diffraction patterns obtained from the Build/Axial and Transverse Directions of the theta specimens for the spot $(a, b)$ and line $(c, d)$ builds. 
Figure 9. 2D neutron diffraction plots from the spot and line builds covering both the build/axial and transverse directions, presented as a function of loading and unloading steps. (axes x: dspacing, y: Cumulative Load $(\mathrm{kN})$, color corresponds to intensity: the brighter the more intense)

Figure 10. The evolution of the lattice strains presented as a function of the applied cumulative loads covering both the axial and transverse directions for a) spot build and b) line build. The evolution of the axial lattice strains as a function of the macroscopic strains experienced by the cross-member for c) spot build and d) line build. The closed symbols correspond to the axial lattice strains whereas the open symbols correspond to the transverse lattice strains. The data points correspond to the average response of five mapped locations on the cross-member (Center, \pm 2 and $\pm 4 \mathrm{~mm}$, Figure $3 \mathrm{~b}$ ) and the error bars to their standard deviation.

Figure 11. A schematic illustrating the iso-strain condition loading of various grains with different crystallographic orientations.

Figure 12. The evolution of the axial and transverse lattice strains presented as a function of the (311) axial stresses on the cross-members of a) spot build and b) line build. The evolution of the transverse lattice strains presented as a function of the (311) axial stresses on the cross-members of c) spot build and d) line build. Also presented in c and d are the calculated/expected responses of the (220) and (420) orientations, for comparison to the experimentally observed values. Here closed symbols correspond to the axial lattice strains, open symbols to the transverse lattice strains and the half-closed symbols to the calculated/expected transverse lattice strains. The arrows mark the overlapping transverse strain behavior of the (200), (220) and (420) reflections. 
Figure 13. A schematic showing the orientation relation of an applied load, $\sigma_{\text {applied, }}$ with respect to the $<001>$ axes of a crystal. 
Table 1. The hkl-specific Poisson's ratios $\left(v_{\mathrm{hkl}}\right)$ presented for the (200), (220), (311) and the (420) orientations from experimental results for both builds (Spot Build: SB and Line Build: LB) in comparison to the ones obtained from the Kröner model from Ref. [39].

\begin{tabular}{|c|c|c|}
\hline hkl & Experimental (SB/LB) & Kröner [39] \\
\hline 200 & $0.372 / 0.386$ & 0.40 \\
\hline 220 & $0.553 / 0.572$ & 0.33 \\
\hline 311 & $0.341 / 0.349$ & 0.36 \\
\hline 420 & $0.433 / 0.438$ & 0.36 \\
\hline
\end{tabular}


a)

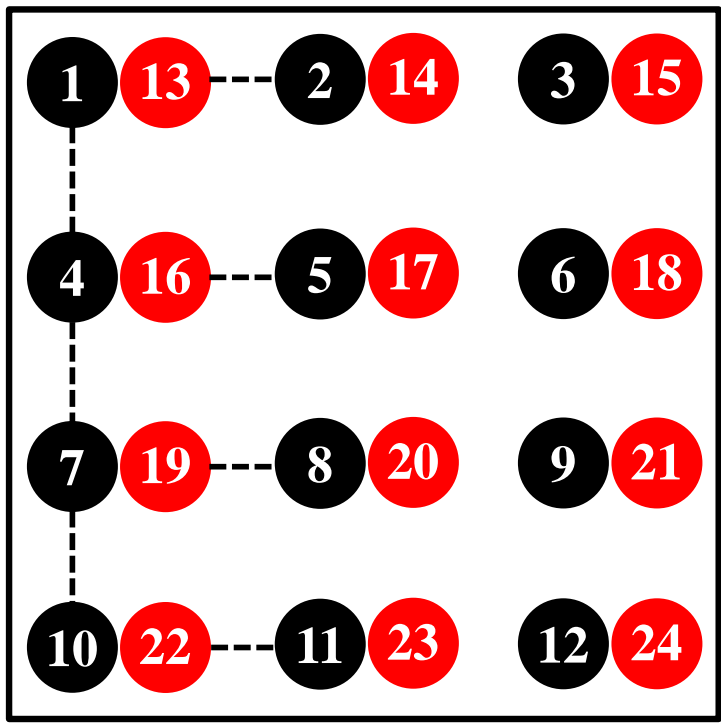

b)

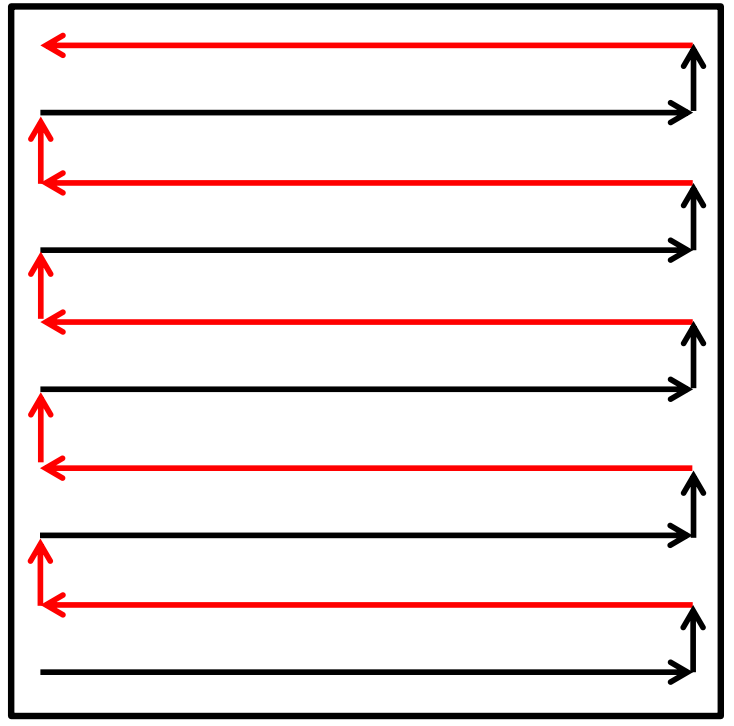

Figure 1. Schematics illustrating the build patterns of the a) Spot and b) Line build strategies. The Spot Build consists of melting individual 'spots' one point at a time in a pattern whereas the Line Build does a continuous melt following a snake raster pattern. 


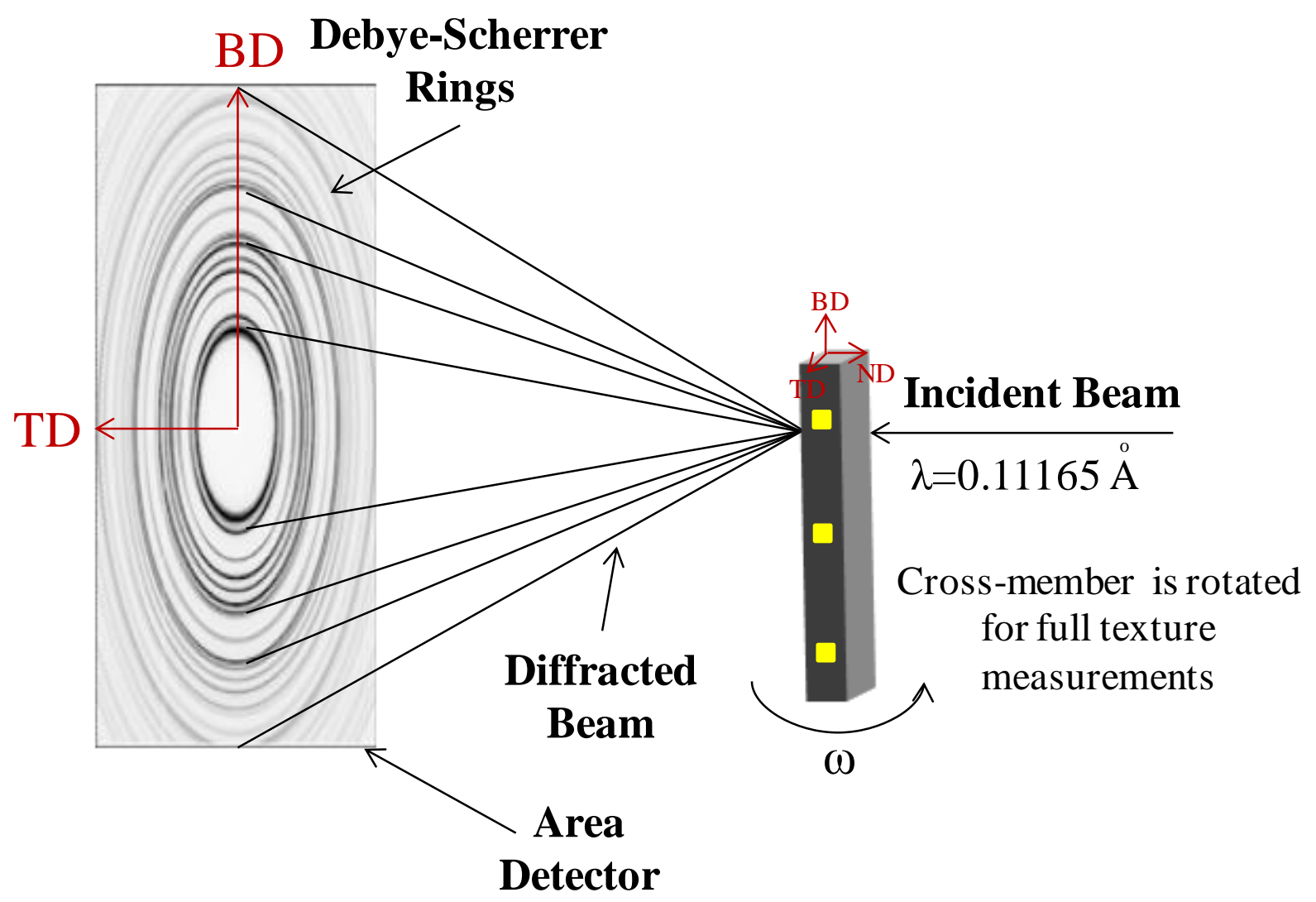

Figure 2. A schematic of the synchrotron x-ray diffraction measurement setup in transmission geometry. BD, TD, and ND refer to the build, transverse, and normal directions of the crossmember, respectively; and $\omega$ corresponds to the rotation axis for the texture measurements. Also shown on the schematic are the locations along the cross-member (see yellow squares) from which the data was obtained. 

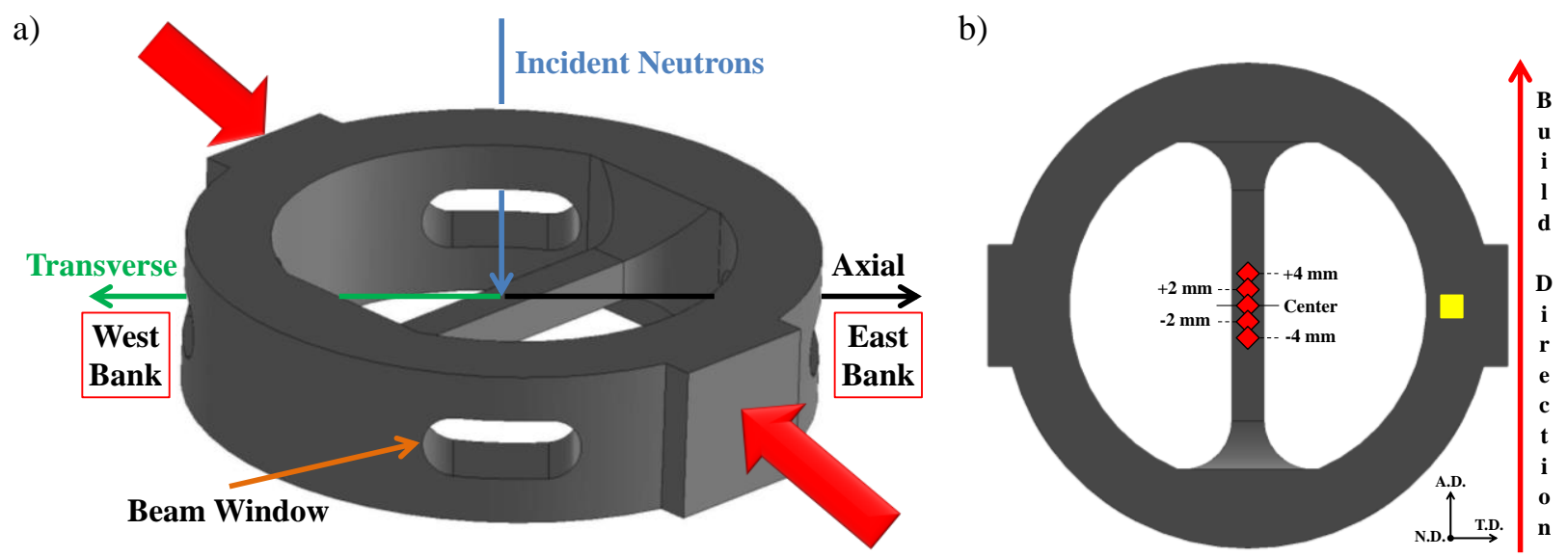

Figure 3. a) A schematic of the in-situ loading neutron diffraction measurement setup. The signal from the planes perpendicular (i.e., their plane normals parallel) to the build/axial direction and along the cross-member is received in the east bank of detectors. Likewise, the signal from the planes perpendicular to the transverse direction is received by the west bank of detectors. The red arrows correspond to the direction of the applied compressive load on the theta sample which places the cross-member in tension. Note the beam "windows" which allow the passage of the incident and diffracted beams to and from the region of interest with minimal attenuation; b) The location of the neutron diffraction mapping points along the cross-member. A.D., T.D., and N.D. refer to the axial, transverse and normal directions, respectively. Also shown here is the location on the ring section (see yellow square) from where the EBSD measurements were taken. 


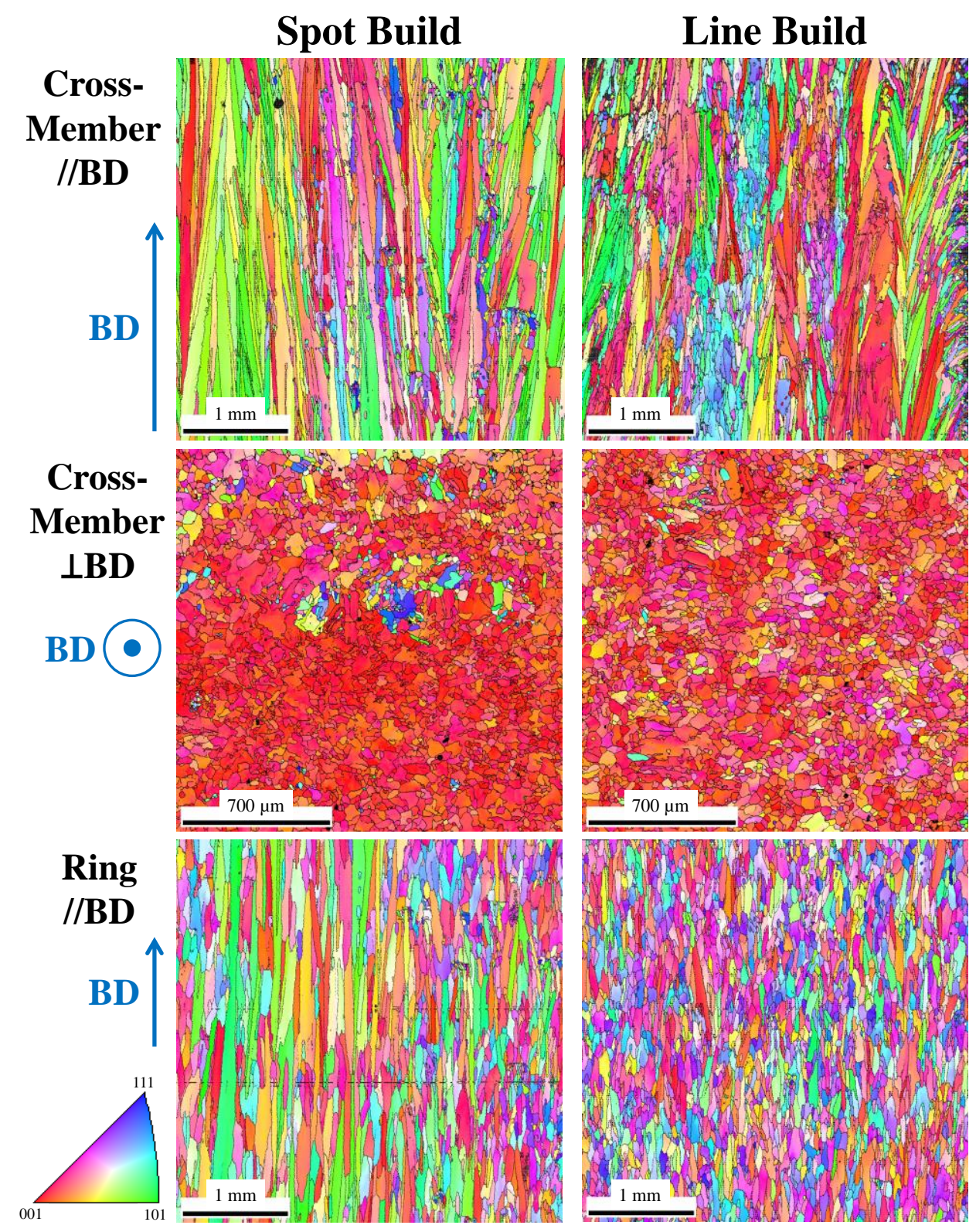

Figure 4. EBSD grain orientation maps obtained from the cross-members parallel (//) and perpendicular $(\perp)$ to the build direction (BD) and from the ring sections parallel (//) to BD; presented for both the spot and the line builds. In the case of $/ / \mathrm{BD}$, the build direction lies along the vertical axis of the micrographs (see the arrow) whereas it is pointing out of the plane of the paper in the case of $\perp$ BD. 


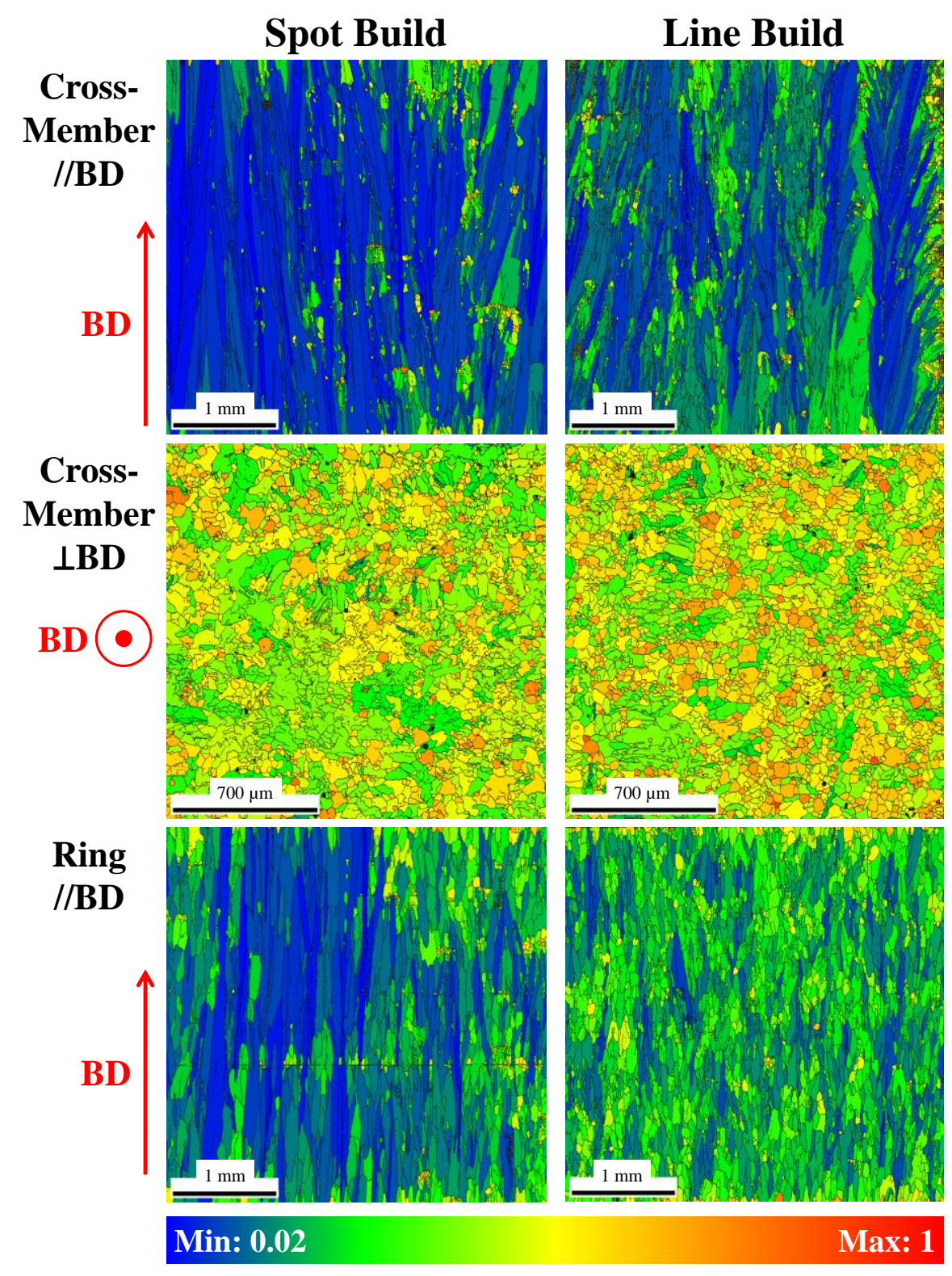

Figure 5. EBSD grain aspect ratio maps obtained from the cross-members parallel (//) and perpendicular $(\perp)$ to the build direction $(\mathrm{BD})$ and from the ring sections parallel $(/ /)$ to $\mathrm{BD}$; presented for both the spot and the line builds. In the case of //BD, the build direction lies along the vertical axis of the micrographs (see the arrow) whereas it is pointing out of the plane of the paper in the case of $\perp \mathrm{BD}$. The warmer colors correspond to more equiaxed grains with red being the most equiaxed and blue being the most columnar. 


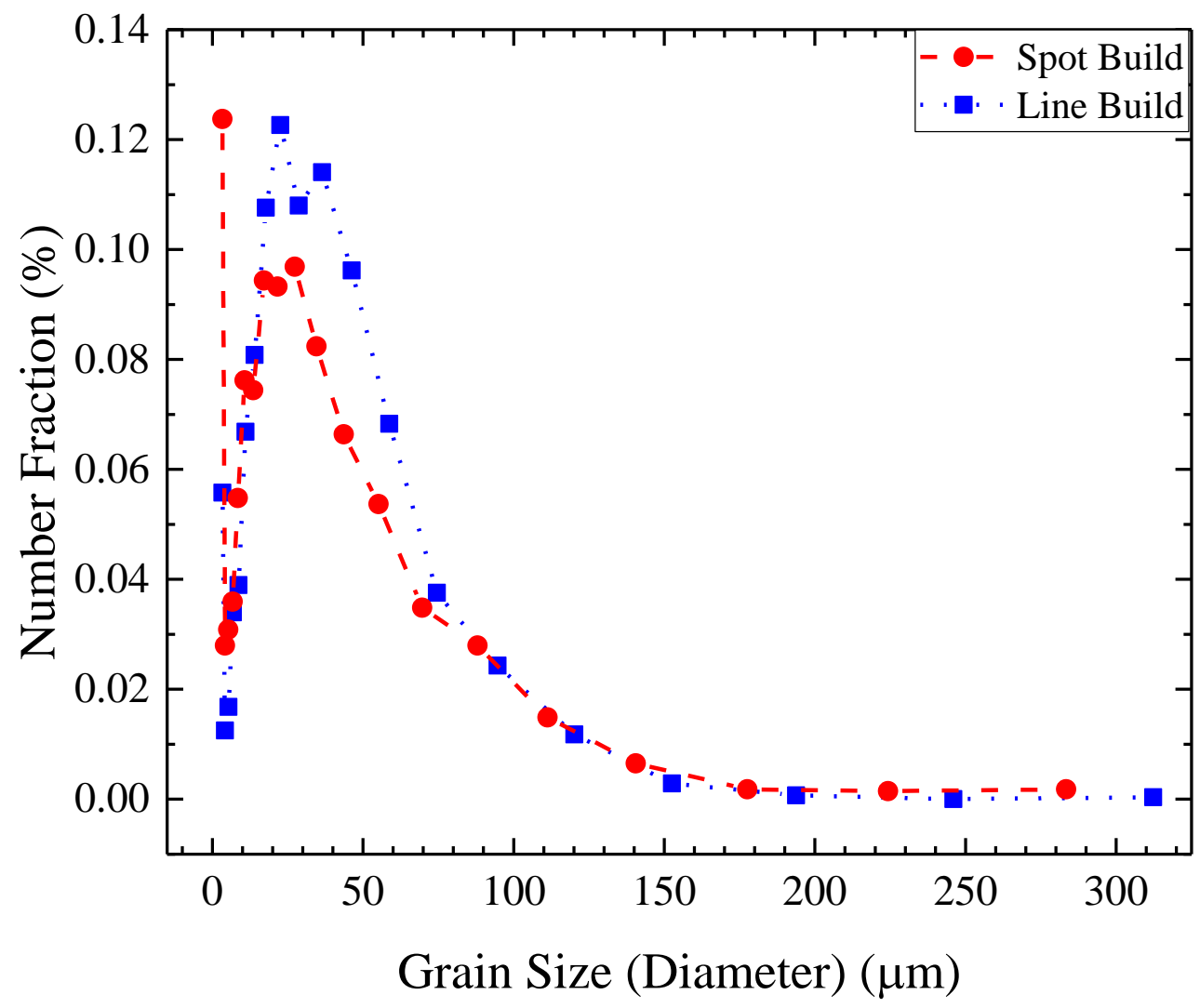

Figure 6. Grain size (Diameter) distributions obtained from the cross-members of both the spot and the line builds, perpendicular to the build direction (see Figures 4 and 5). 
a)
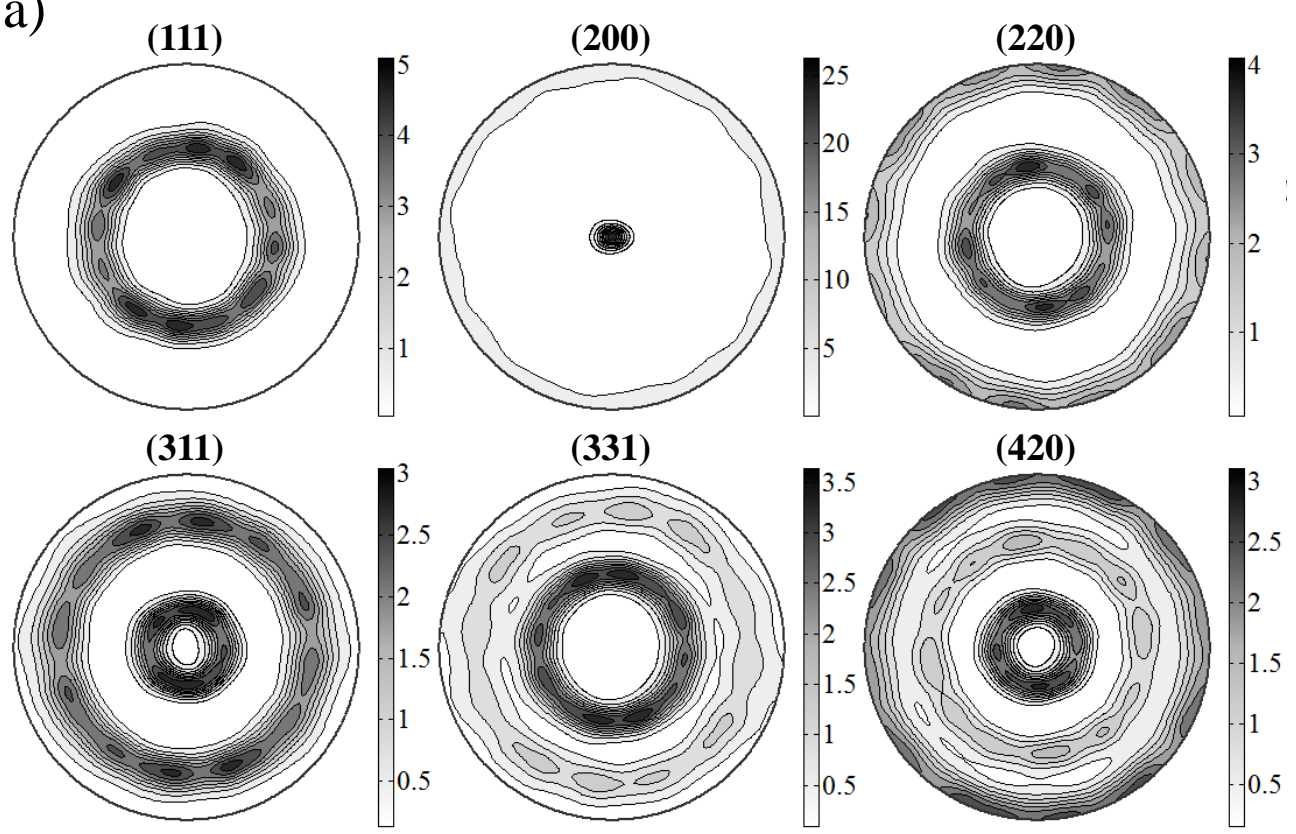

b)
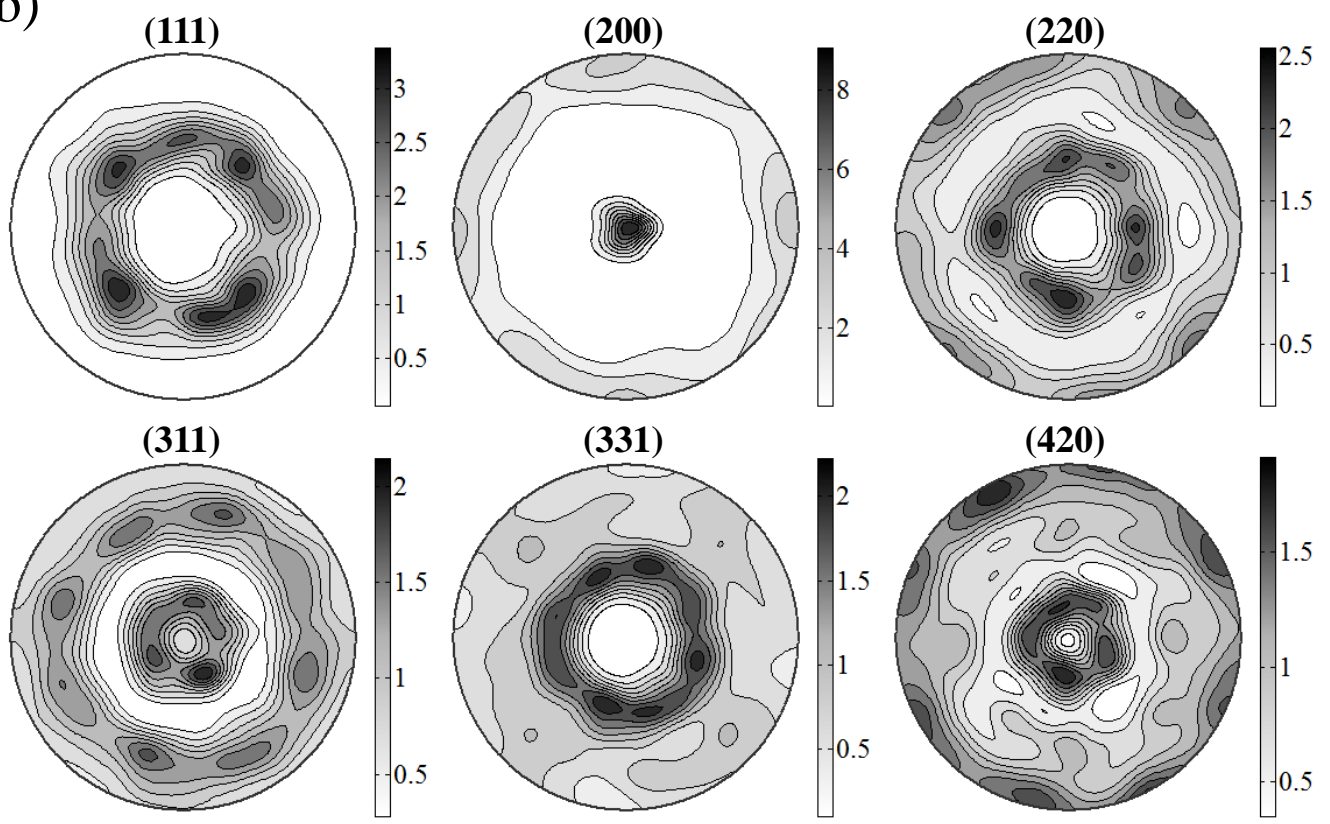

Figure 7. (111), (200), (220), (311), (331) and (420) pole figures for a) Spot Build and b) Line Build obtained with SXRD. The Build Direction is at the center of the pole figures pointing out of the plane of the paper. The darker colors represent higher levels of intensity, and the intensity levels are on linear scale. 
a)

\section{Build/Axial Direction}

Spot Build

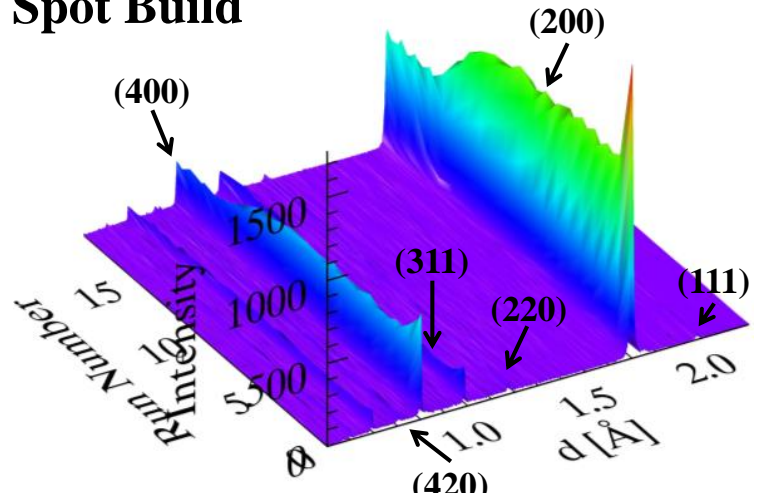

c)

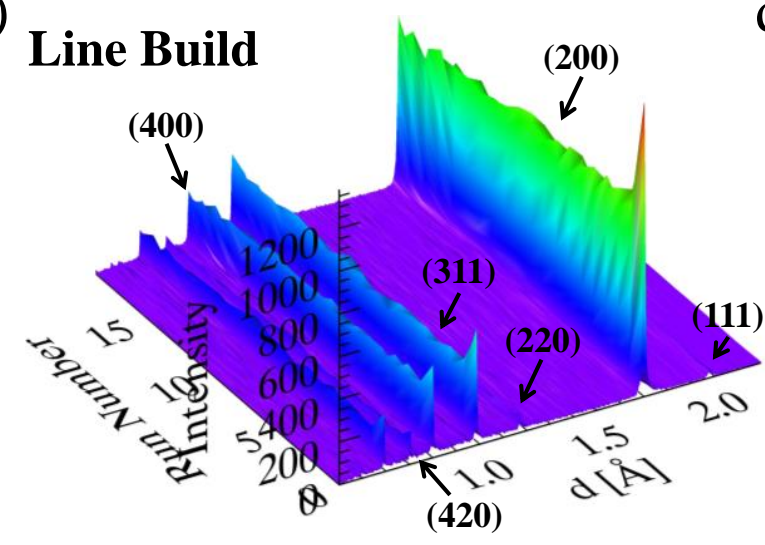

b)

\section{Transverse Direction}

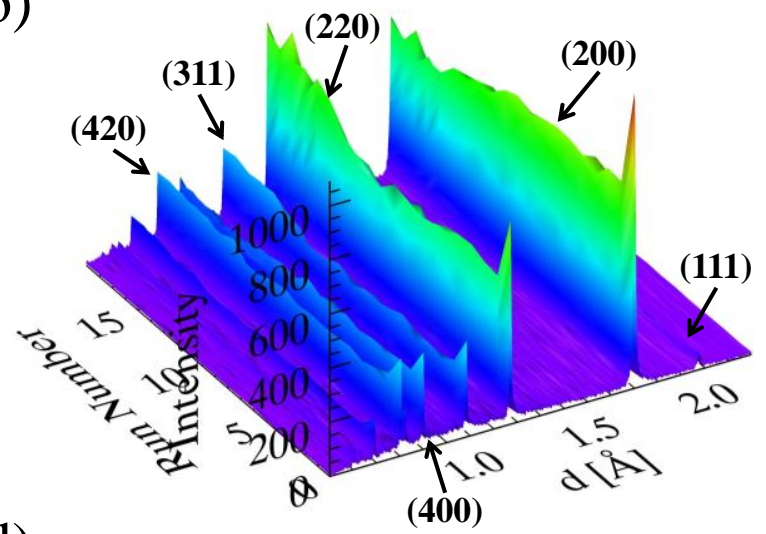

d)

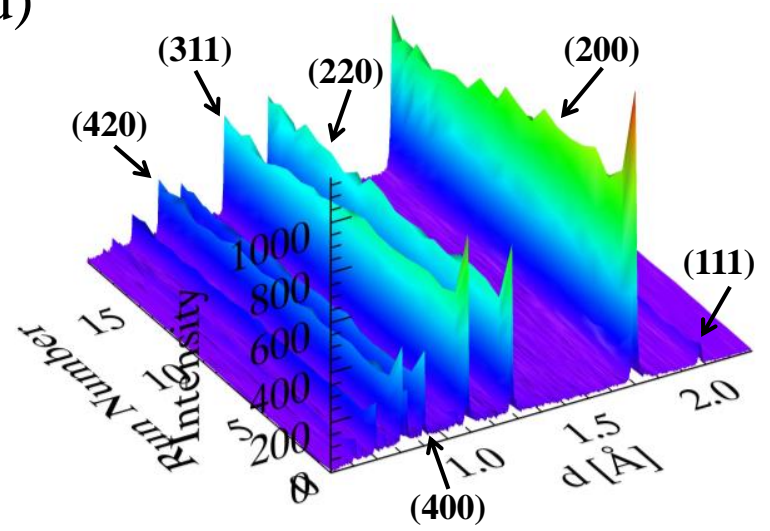

Figure 8. Neutron diffraction patterns obtained from the Build/Axial and Transverse Directions of the theta specimens for the spot $(\mathrm{a}, \mathrm{b})$ and line $(\mathrm{c}, \mathrm{d})$ builds. 

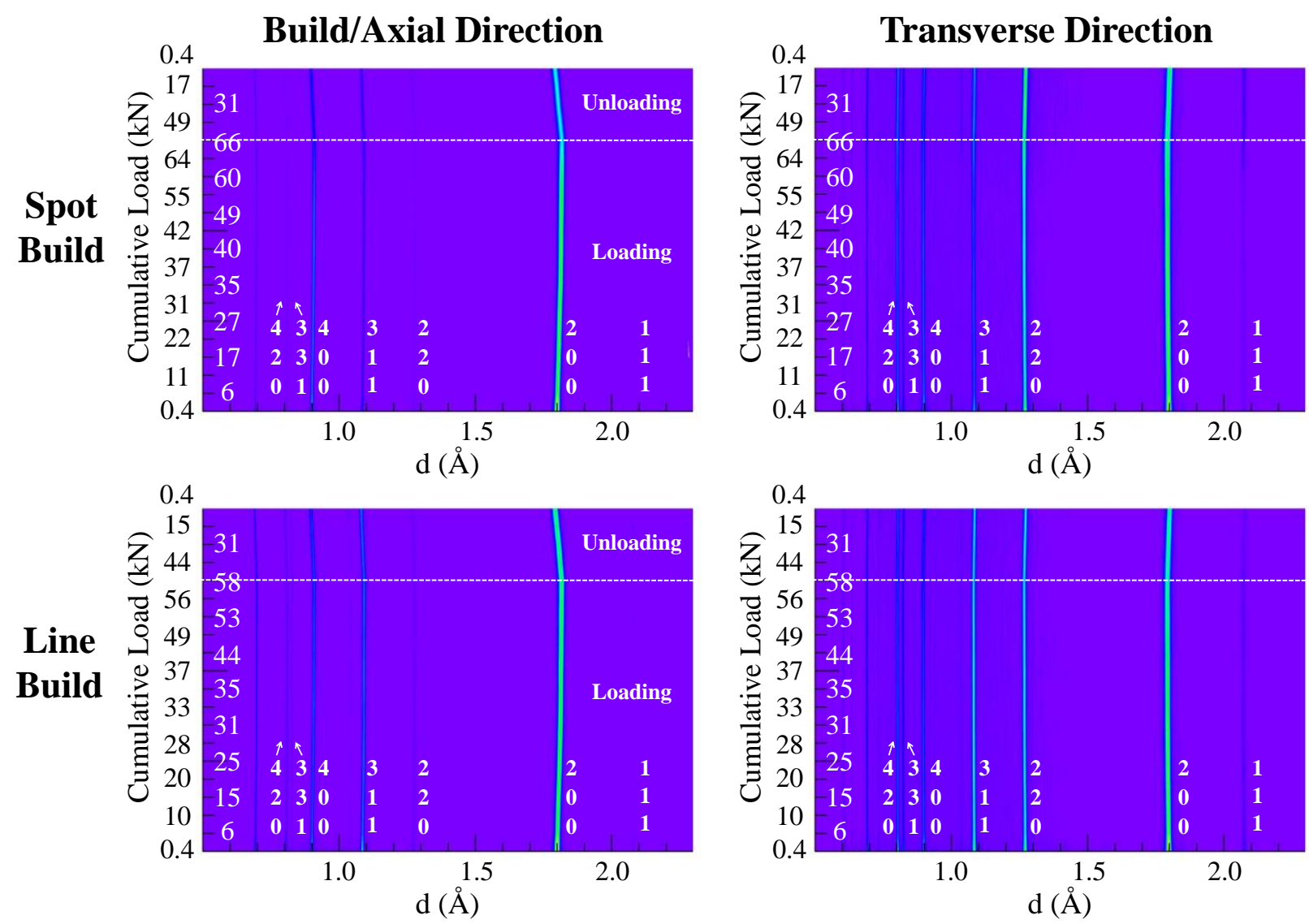

Figure 9. 2D neutron diffraction plots from the spot and line builds covering both the build/axial and transverse directions, presented as a function of loading and unloading steps. (axes x: dspacing, y: Cumulative Load $(\mathrm{kN})$, color corresponds to intensity: the brighter the more intense) 
a)

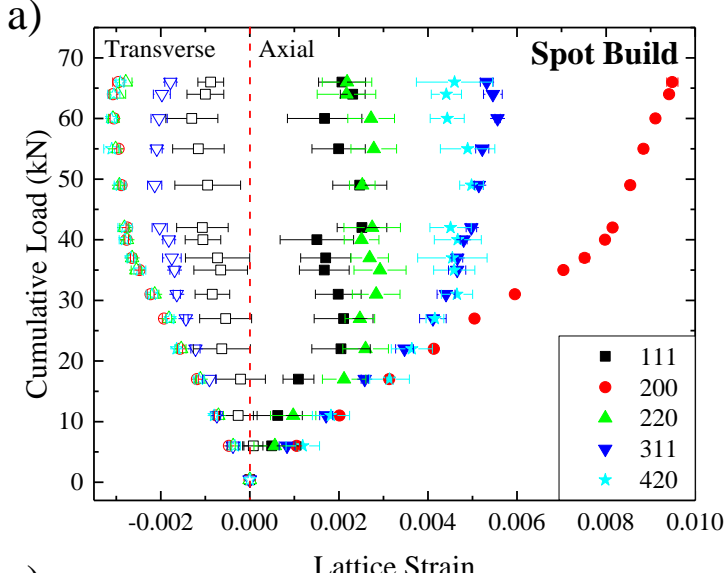

c)

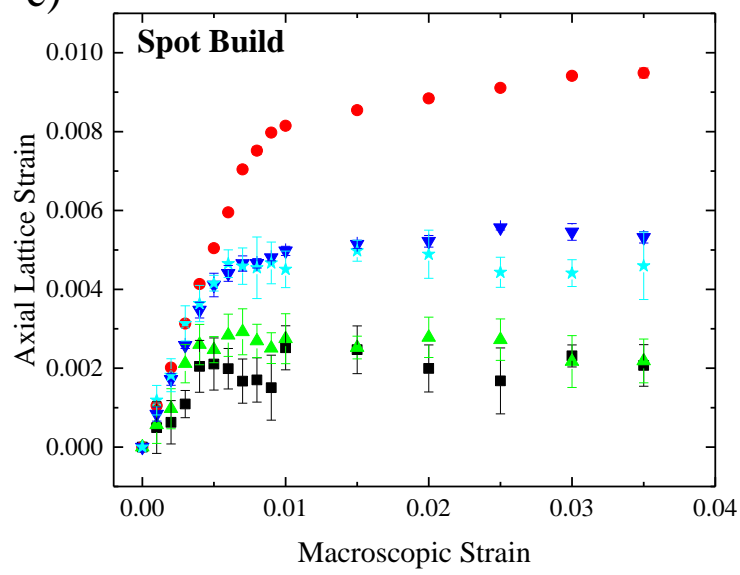

b)

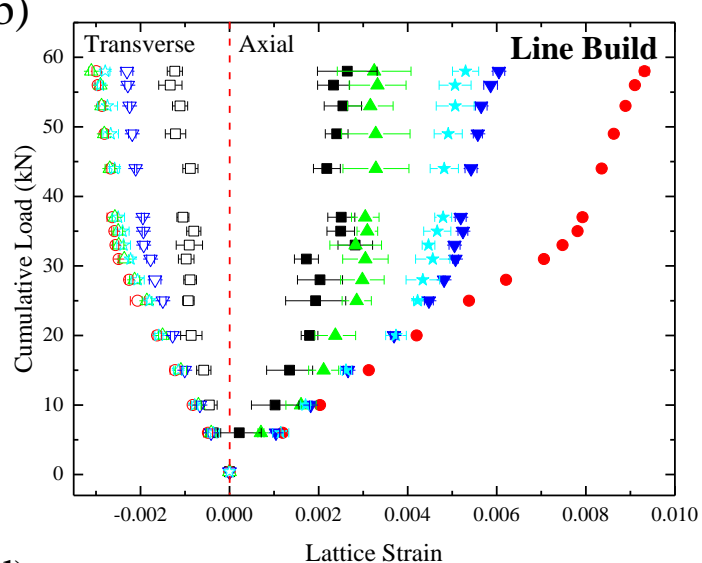

d)

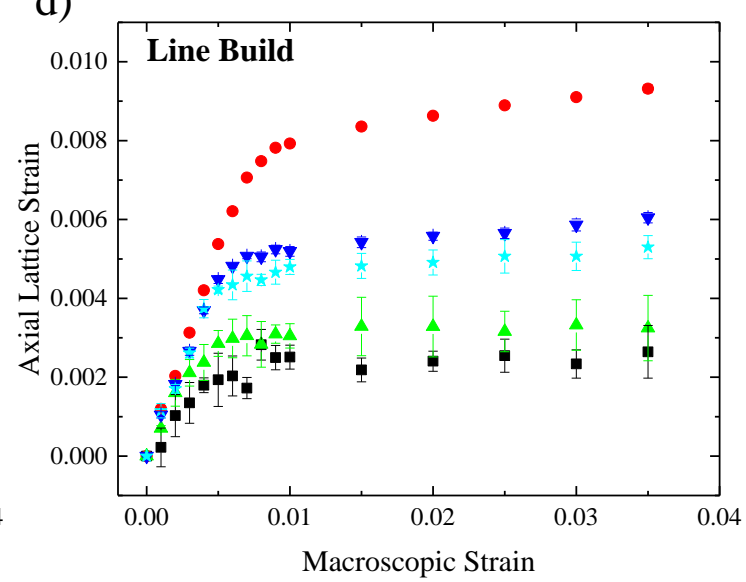

Figure 10. The evolution of the lattice strains presented as a function of the applied cumulative loads covering both the axial and transverse directions for a) spot build and b) line build. The evolution of the axial lattice strains as a function of the macroscopic strains experienced by the cross-member for c) spot build and d) line build. The closed symbols correspond to the axial lattice strains whereas the open symbols correspond to the transverse lattice strains. The data points correspond to the average response of five mapped locations on the cross-member (Center, \pm 2 and $\pm 4 \mathrm{~mm}$, Figure $3 \mathrm{~b}$ ) and the error bars to their standard deviation. 


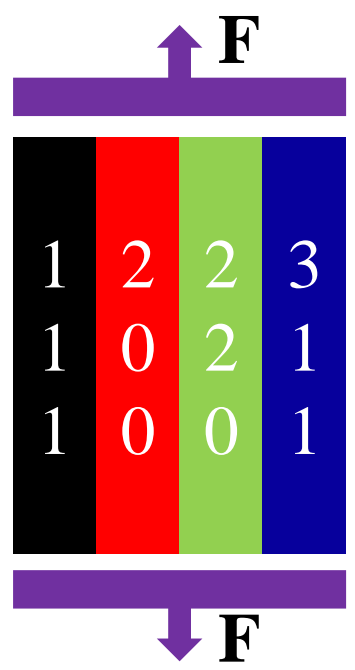

Figure 11. A schematic illustrating the iso-strain condition loading of various grains with different crystallographic orientations. 
a)

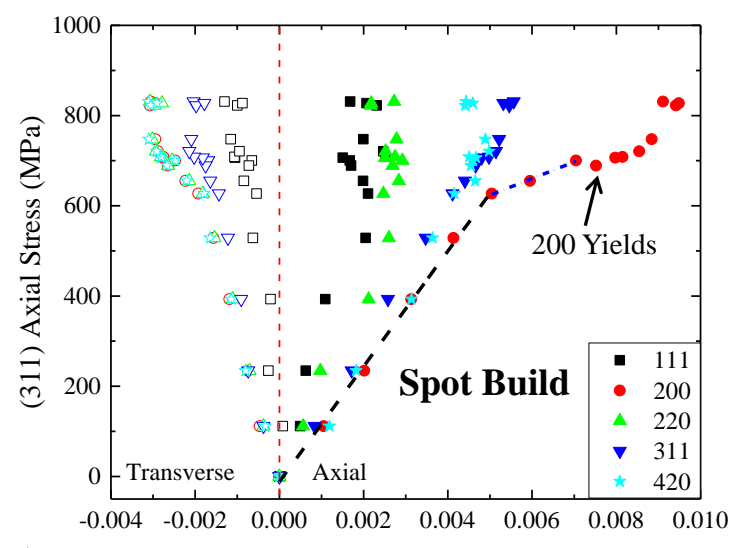

c)

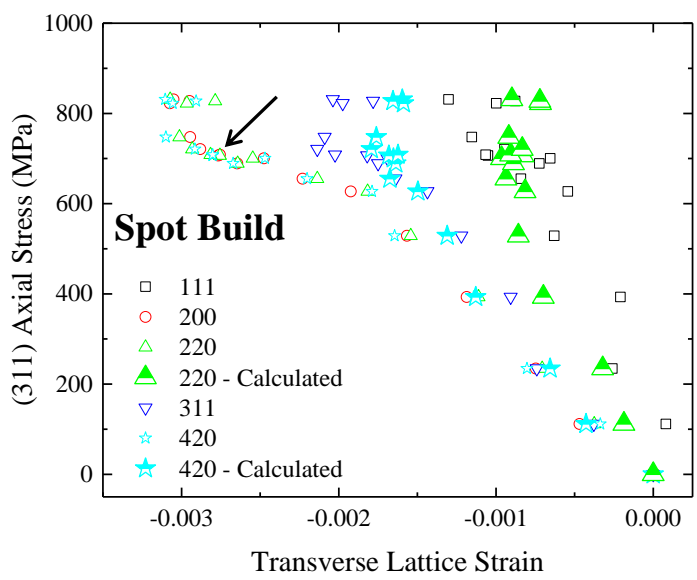

b)

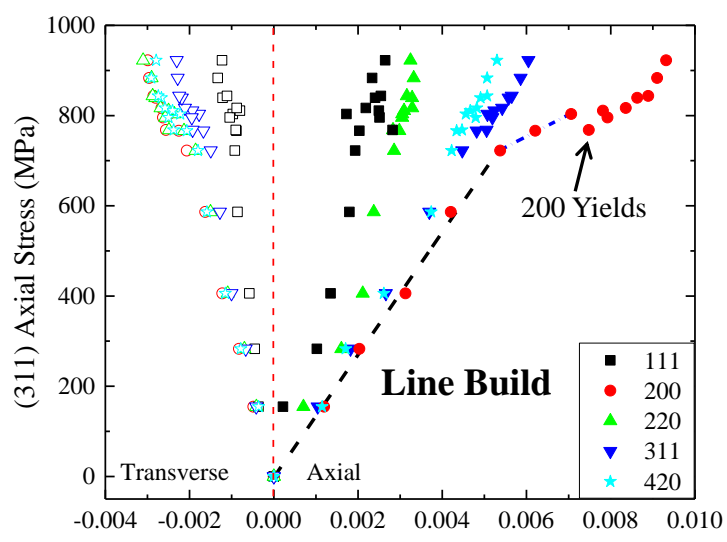

d)

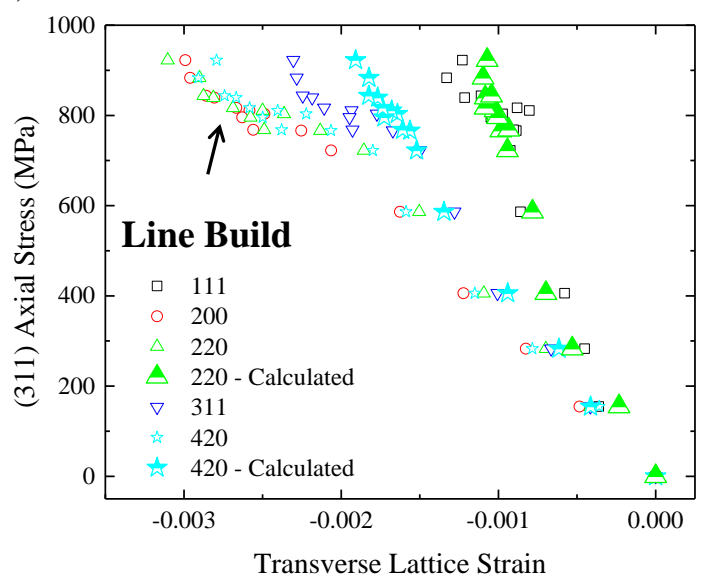

Figure 12. The evolution of the axial and transverse lattice strains presented as a function of the (311) axial stresses on the cross-members of a) spot build and b) line build. The evolution of the transverse lattice strains presented as a function of the (311) axial stresses on the cross-members of c) spot build and d) line build. Also presented in c and d are the calculated/expected responses of the (220) and (420) orientations, for comparison to the experimentally observed values. Here closed symbols correspond to the axial lattice strains, open symbols to the transverse lattice strains and the half-closed symbols to the calculated/expected transverse lattice strains. The arrows mark the overlapping transverse strain behavior of the (200), (220) and (420) reflections. 


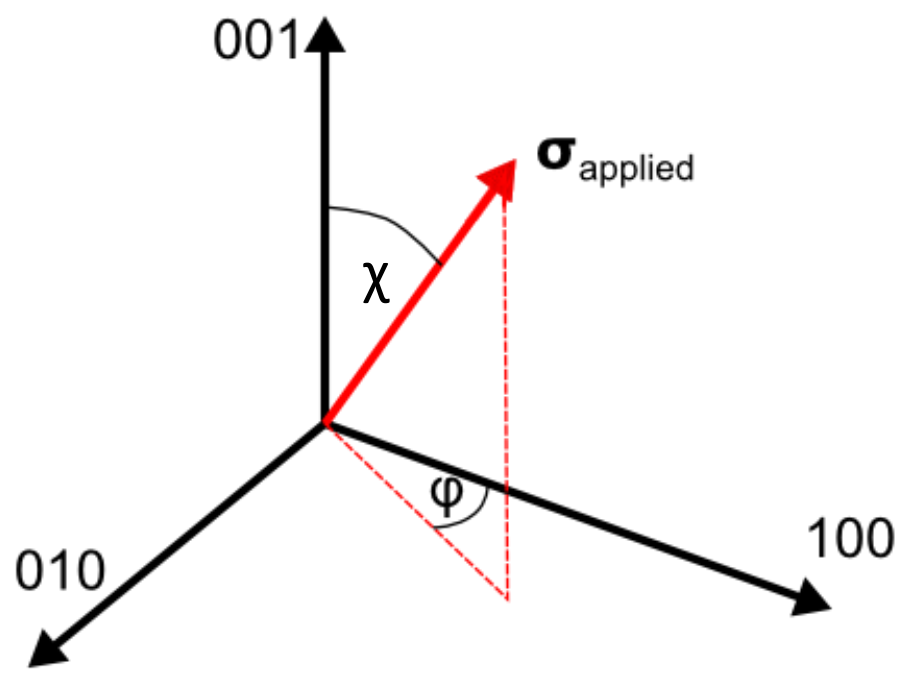

Figure 13. A schematic showing the orientation relation of an applied load, $\sigma_{\text {applied, }}$, with respect to the $<001>$ axes of a crystal. 

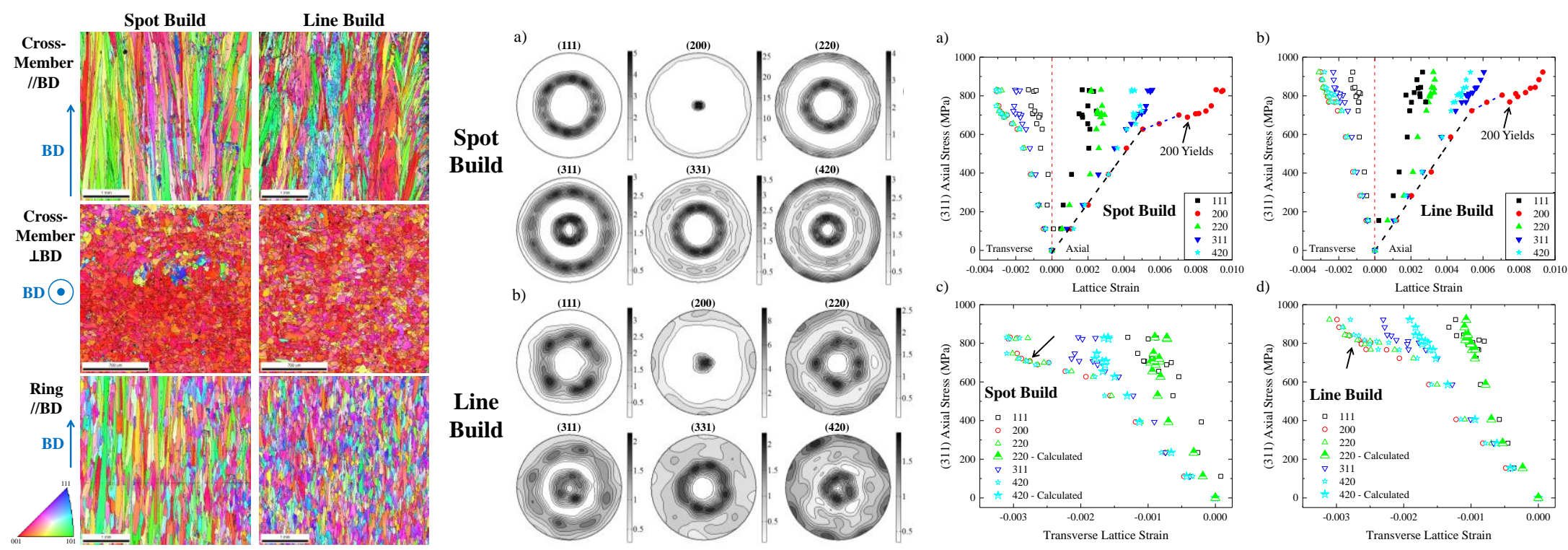

Graphical Abstract 